\title{
Elementary charge-transfer processes in mesoscopic conductors
}

\author{
Mihajlo Vanević, ${ }^{1}$ Yuli V. Nazarov, ${ }^{2}$ and Wolfgang Belzig ${ }^{3}$ \\ ${ }^{1}$ Departement Physik, Universität Basel, Klingelbergstrasse 82, CH-4056 Basel, Switzerland \\ ${ }^{2}$ Kavli Institute of Nanoscience, Delft University of Technology, 2628 CJ Delft, The Netherlands \\ ${ }^{3}$ Fachbereich Physik, Universität Konstanz, D-78457 Konstanz, Germany
}

(Received 22 September 2008; published 8 December 2008)

\begin{abstract}
We determine charge-transfer statistics in a quantum conductor driven by a time-dependent voltage and identify the elementary transport processes. At zero temperature unidirectional and bidirectional single-charge transfers occur. The unidirectional processes involve electrons injected from the source terminal due to excess dc bias voltage. The bidirectional processes involve electron-hole pairs created by time-dependent voltage bias. This interpretation is further supported by the charge-transfer statistics in a multiterminal beam-splitter geometry in which injected electrons and holes can be partitioned into different outgoing terminals. The probabilities of elementary processes can be probed by noise measurements: the unidirectional processes set the dc noise level, while bidirectional ones give rise to the excess noise. For ac voltage drive, the noise oscillates with increasing the driving amplitude. The decomposition of the noise into the contributions of elementary processes reveals the origin of these oscillations: the number of electron-hole pairs generated per cycle increases with increasing the amplitude. The decomposition of the noise into elementary processes is studied for different time-dependent voltages. The method we use is also suitable for systematic calculation of higher-order current correlators at finite temperature. We obtain current noise power and the third cumulant in the presence of time-dependent voltage drive. The charge-transfer statistics at finite temperature can be interpreted in terms of multiple-charge transfers with probabilities which depend on energy and temperature.
\end{abstract}

DOI: 10.1103/PhysRevB.78.245308 PACS number(s): 73.23. $-\mathrm{b}, 72.70 .+\mathrm{m}, 72.10 . \mathrm{Bg}, 05.40 .-\mathrm{a}$

\section{INTRODUCTION}

The charge transmitted through a mesoscopic conductor during a fixed time interval fluctuates because of charge discreteness and stochastic nature of transport. The objective of the statistical theory of quantum transport, full counting statistics, is to completely characterize the probability distribution of transferred charge. The field of full counting statistics has attracted significant attention because it provides the most detailed information on charge transfer accessible in the measurements of the average current, current noise power, ${ }^{1}$ and higher-order current correlations. ${ }^{2-4}$

The problem of full counting statistics has been addressed first by Levitov and Lesovik ${ }^{5,6}$ for the case of dc biased multiterminal junctions and subsequently generalized to a time-dependent voltage bias. ${ }^{7,8}$ In addition to the scattering theory of Levitov and Lesovik ${ }^{5,6}$ (see also Ref. 9), the theoretical approaches to full counting statistics include the socalled stochastic path-integral approach ${ }^{10,11}$ and the quantum-mechanical theory based on an extension of the Keldysh-Green's-function technique. ${ }^{12-16}$ Although equivalent, these theories provide different methods to access the charge-transfer statistics. Using the extended KeldyshGreen's-function technique, the theory of full counting statistics of a general quantum-mechanical observable has been formulated, ${ }^{17,18}$ including the effects of a detector back action. ${ }^{19}$ The problem of quantum measurement in the context of full counting statistics of noncommuting spin observables has also been analyzed. ${ }^{20,21}$ The discretization ${ }^{22,23}$ in space of the Keldysh-Green's functions results in a quantum circuit theory ${ }^{24-26}$ which greatly simplifies the calculations. The circuit theory can describe junctions with different connectors and leads, as well as multiterminal circuits. ${ }^{27}$
The goal of evaluation of full counting statistics in mesoscopic conductors has essentially been accomplished: the aforementioned theories provide methods and techniques to calculate the cumulant generating function of transferred charge from which the probability distribution can be obtained. However, the interpretation of the resulting statistics is often not straightforward. This is because the total statistics are composed of many electrons injected toward conductor, each exhibiting different chaotic scattering and multiple reflections before entering an outgoing terminal. At finite temperatures, the charge flow is bidirectional with both injection and absorption of electrons from terminals. The particle correlations due to Pauli principle, interactions, and those induced by the superconducting and/or ferromagnetic proximity effect also affect the charge-transfer statistics.

To understand the statistical properties of collective charge transfer, one has to find the independent elementary processes constituting the statistics. The independent processes can be revealed by decomposition of the total cumulant generating function into a sum of simpler ones. The physical interpretation obtained this way goes beyond information contained in the average current, noise, and finiteorder cumulants and pertains to all transport measurements.

The elementary processes in a dc biased normal junction are single-electron transfers, which are independent at different energies. At finite temperatures, the electrons are transferred in both directions with correlated left and right transfers. At low temperatures only unidirectional electron transfers remain, with direction set by polarity of applied voltage. The elementary processes in a superconductor- normal contact are single- and double-charge transfers. ${ }^{24,28}$ At low temperatures and voltages below the superconducting gap only double-charge Andreev processes remain, while above the gap normal single-charge transfers dominate. The 
elementary charge-transfer processes between superconductors with dc bias applied consist of multiple-charge transfers due to multiple Andreev reflections. ${ }^{29-32}$ The interpretation of elementary charge-transfer processes between superconductors with constant phase difference is more subtle. In this case charge transfers acquire formally negative probabilities and the proper physical interpretation has to include dynamics of a detector. ${ }^{13,17,18}$

In contrast to dc voltage bias, time-dependent voltage drive mixes electron states of different energies which in combination with the Pauli principle leads to a nontrivial charge-transfer statistics. The signatures of this statistics have been studied first through the noise in an ac-driven junction (photon-assisted noise). ${ }^{33,34}$ The photon-assisted noise at low temperatures is a piecewise linear function of a dc voltage offset with kinks corresponding to integer multiples of the driving frequency and slopes which depend on the amplitude and shape of the ac component. This dependence has been observed experimentally in normal coherent conductors ${ }^{35,36}$ and diffusive normal-metal-superconductor junctions. ${ }^{37}$ The photon-assisted noise at small driving amplitudes is due to an electron-hole pair which is created by ac drive (with a low probability per voltage cycle) and injected toward the scatterer. ${ }^{38-40}$ However, to obtain the elementary processes, the knowledge of full charge-transfer statistics is needed.

The elementary processes in the presence of timedependent drive have been identified in Refs. 41-43 for the special choice of a driving voltage. The authors have studied Lorentzian voltage pulses of the same sign carrying integer numbers of charge quanta. In this case the elementary processes are electrons injected toward the scatterer without creation of the electron-hole pairs. The charge transfer is unidirectional with binomial statistics set by the effective dc voltage. This result is independent of the relative position of the pulses, their duration, and overlap. The many-body quantum state generated by these pulses has been obtained recently. ${ }^{43}$ An alternative way to inject single electrons free of electron-hole pairs is by using time-dependent shifts of a resonant level in a quantum dot. ${ }^{44-48}$

The elementary charge-transfer processes for arbitrary time-dependent voltage drive have been identified in Ref. 49. At low temperatures these processes are single-charge transfers which originate from electrons and electron-hole pairs injected toward the scatterer. The electrons are injected due to excess dc voltage applied and give a binomial contribution to the total cumulant generating function. The electron-hole pairs are created by the ac component of the voltage drive. The probabilities of pair creations per voltage cycle depend on the details of the ac drive. For the special choice of optimal Lorentzian voltage pulses no electron-hole pairs are created. In general, however, an ac drive does create electronhole pairs, with more and more pairs created per voltage cycle as the amplitude of the drive increases. A geometric interpretation of elementary processes is studied in Ref. 50. For time-dependent scatterer, the constraints imposed on charge-transfer statistics restrict the allowed charge transfers. ${ }^{51}$

In this paper we present a comprehensive study of elementary processes in a generic mesoscopic conductor in

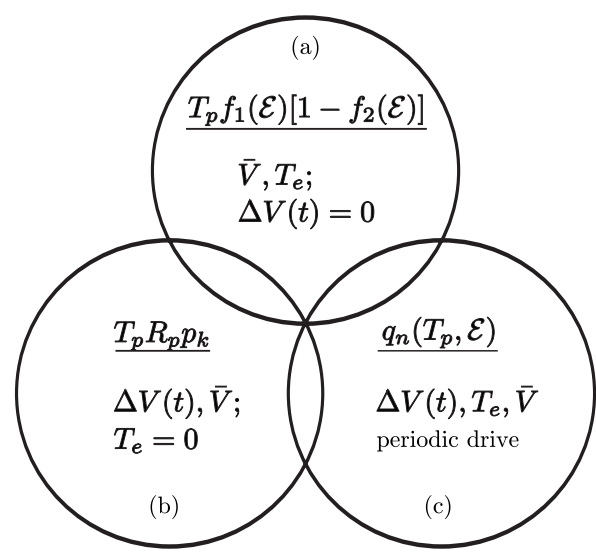

FIG. 1. The elementary processes shown in different regimes. (a) For a dc bias voltage $\bar{V}$ and at arbitrary temperature $T_{e}$, the electrons are transferred independently at different energies. The probability of transfer is given by occupation numbers $f_{1,2}(\mathcal{E})$ of the leads and transmissions $\left\{T_{p}\right\}$ of the junction. The transport occurs when the incoming electronic state is occupied, the outgoing state is empty, and the electron is transferred across the junction. (b) On the other hand, an ac voltage drive $\Delta V(t)$ mixes the electron states of different energies. At zero temperature it creates the electron-hole pairs with probabilities $p_{k}$, in addition to electrons injected due to excess dc voltage. A created electron-hole pair contributes to transport only if one particle from a pair is transmitted and the other is reflected, which occurs with probability $T_{p} R_{p}\left(R_{p}\right.$ being reflection coefficient). (c) Finally, at finite temperature and in the presence of a time-dependent voltage drive, the statistics can be interpreted in terms of multiple $n$-charge transfers with probabilities $q_{n}$.

different physical regimes, as depicted in Fig. 1. We first obtain the cumulant generating function for a coherent voltage-driven quantum contact at finite temperatures, using both the formalism of Levitov and Lesovik ${ }^{5,6}$ and the circuit theory of quantum transport (Sec. II). We then identify the elementary charge-transfer processes at zero temperature (Sec. III A). Decomposition of the noise into contributions of elementary processes for different voltage signals is studied in Sec. III B.

For ac voltage drive, the differential noise oscillates as the amplitude of the drive increases due to new electron-hole pairs being created per voltage cycle. The number of electron-hole pairs per cycle becomes large for driving amplitudes much larger than frequency. In this case the statistics in the leading order reduces to uncorrelated electron and hole transfers and depends on an effective voltage only, independent of the details of the ac drive. The elementary processes can be probed also for both ac and dc voltages present, e.g., in the regime in which the ac component of the drive is kept fixed and the dc offset changes. In this case both the electrons and created electron-hole pairs are injected toward the junction. The electron-hole pairs give rise to the excess noise with respect to the noise level set by dc voltage offset.

The notion of elementary processes, being a matter of interpretation of the full charge-transfer statistics, provides physically plausible and intuitive description of transport in terms of statistically independent events. Such a description persists in all transport measurements. For example, the picture of electrons and electron-hole pairs injected toward the 
scatterer, which we have inferred from a study of a twoterminal junction, is further supported by the evaluation of the full counting statistics in a beam-splitter geometry in which the injected particles can be separated into different outgoing terminals. These processes can be probed by current cross correlations (Sec. III C).

The method we use is also suitable for systematic calculation of the higher-order current correlators at finite temperature. The current noise power and the third cumulant in the presence of time-dependent voltage drive are obtained in Sec. IV. The interpretation of the full counting statistics and the elementary processes are fundamentally different at finite temperature. For a periodic drive, the elementary processes are transfers of multiple integer charge quanta with probabilities which depend on energy and temperature (Sec. V).

\section{CUMULANT GENERATING FUNCTION}

The system we consider is a generic two-terminal mesoscopic conductor characterized by a set of transmission eigenvalues $\left\{T_{p}\right\}$. For a large number of channels, the transport properties are universal and independent of microscopic details of geometry of the junction and positions of impurities. We can neglect the energy dependence of transmission eigenvalues if the electron dwell time is small with respect to time scales set by the inverse temperature and applied voltage. The cumulant generating function of the transferred charge is given by determinant formula $5,41,52$

$$
\mathcal{S}(\chi)=\ln \operatorname{det}\left[1+\mathbf{f}\left(\mathbf{S}^{\dagger} \boldsymbol{\Lambda}_{\chi}^{\dagger} \mathbf{S} \boldsymbol{\Lambda}_{\chi}-1\right)\right]
$$

where

$$
\mathbf{f}=\left(\begin{array}{cc}
f_{1} & 0 \\
0 & f_{2}
\end{array}\right), \quad \mathbf{S}=\left(\begin{array}{cc}
\mathbf{r} & \mathbf{t}^{\prime} \\
\mathbf{t} & \mathbf{r}^{\prime}
\end{array}\right), \quad \boldsymbol{\Lambda}_{\chi}=\left(\begin{array}{cc}
e^{-i \chi} & 0 \\
0 & 1
\end{array}\right) .
$$

Here $\mathbf{f}$ is the matrix of occupation numbers of the terminals which is diagonal in the terminal indices and scalar in transport channels, $\mathbf{S}$ is the scattering matrix of the junction, and $\Lambda_{\chi}^{\dagger} \ldots \Lambda_{\chi}$ is the transformation which incorporates the counting fields. Because the current is conserved, it is sufficient to assign only one counting field $\chi$ to the left terminal. We count charges which enter the left terminal irrespective the channel, the energy, or the spin, with $\chi$ being scalar in the corresponding indices. The determinant in Eq. (1) is taken with respect to the terminal, channel, energy, and spin indices.

The occupation numbers $f_{1}$ and $f_{2}$ of the left and right terminals are matrices in energy indices and scalars in channel and spin indices. In the case of dc voltage bias $f_{1,2}$ are diagonal in energy with $f_{i}\left(\mathcal{E}^{\prime}, \mathcal{E}^{\prime \prime}\right)=f_{i}\left(\mathcal{E}^{\prime}\right) 2 \pi \delta\left(\mathcal{E}^{\prime}-\mathcal{E}^{\prime \prime}\right)$, where $f_{i}(\mathcal{E})=\left(e^{\left(\mathcal{E}-e V_{i}\right) / T_{e}}+1\right)^{-1}, V_{i}$ is the voltage applied, and $T_{e}$ is the electronic temperature. In the presence of timedependent drive the occupation numbers $f_{i}\left(\mathcal{E}^{\prime}, \mathcal{E}^{\prime \prime}\right)$ are not diagonal in energy indices and do not commute. The voltage drive $V(t)$ can be incorporated via the gauge transformation in time representation $f_{1} \rightarrow U f_{V=0} U^{\dagger}$ with $U\left(t^{\prime}, t^{\prime \prime}\right)$ $=\exp \left[-i \int_{0}^{t^{\prime}} e V(t) d t\right] \delta\left(t^{\prime}-t^{\prime \prime}\right)$, where we assume the convolution over internal time indices.

Equation (1) can be simplified using polar decomposition of the scattering matrix, ${ }^{53}$

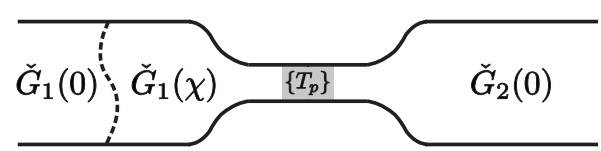

FIG. 2. The schematic circuit-theory representation of a twoterminal junction. The left and right terminals are characterized by the quasiclassical Keldysh-Green's functions $\breve{G}_{1,2}(0)$. The counting field $\chi$ is related to the charges which enter the left terminal through the cross section indicated by dashed line. It can be incorporated into the corresponding Keldysh-Green's function via gauge transformation given by Eq. (8).

$$
\mathbf{S}=\left(\begin{array}{cc}
\mathbf{U} & \mathbf{0} \\
\mathbf{0} & \mathbf{V}
\end{array}\right) \mathbf{S}^{\prime}\left(\begin{array}{cc}
\mathbf{U}^{\prime} & \mathbf{0} \\
\mathbf{0} & \mathbf{V}^{\prime}
\end{array}\right), \quad \mathbf{S}^{\prime}=\left(\begin{array}{cc}
-\sqrt{\mathbf{R}} & \sqrt{\mathbf{T}} \\
\sqrt{\mathbf{T}} & \sqrt{\mathbf{R}}
\end{array}\right) .
$$

Here $\mathbf{U}, \mathbf{V}, \mathbf{U}^{\prime}$, and $\mathbf{V}^{\prime}$ are unitary matrices in transport channels and $\mathbf{T}=\operatorname{diag}\left(T_{1}, T_{2}, \ldots\right)$ and $\mathbf{R}=\mathbf{1}-\mathbf{T}$ are diagonal matrices of transmission and reflection eigenvalues. Equation (1) for the two-terminal junction reduces to

$$
\begin{aligned}
\mathcal{S}(\chi) & =2_{s} \ln \operatorname{det}\left[1+\mathbf{f}\left(\mathbf{S}^{\prime} \boldsymbol{\Lambda}^{\dagger} \mathbf{S}^{\prime} \boldsymbol{\Lambda}-\mathbf{1}\right)\right] \\
& =2_{s} \ln \operatorname{det}\left(\begin{array}{cc}
1+f_{1} \mathbf{T}\left(e^{-i \chi}-1\right) & -f_{1} \sqrt{\mathbf{T R}}\left(e^{i \chi}-1\right) \\
f_{2} \sqrt{\mathbf{T R}}\left(e^{-i \chi}-1\right) & 1+f_{2} \mathbf{T}\left(e^{i \chi}-1\right)
\end{array}\right),
\end{aligned}
$$

where $2_{s}$ takes into account the spin degree of freedom. Since the operators in the first (the second) row commute, the determinant can be calculated blockwise. Using Eq. (A1) given in Appendix we obtain

$$
\begin{aligned}
\mathcal{S}(\chi)= & 2_{s} \sum_{p} \operatorname{Tr}_{\mathcal{E}} \ln \left[1+\left(1-f_{1}\right) f_{2} T_{p}\left(e^{i \chi}-1\right)\right. \\
& \left.+f_{1}\left(1-f_{2}\right) T_{p}\left(e^{-i \chi}-1\right)\right] \\
= & 2_{s} \sum_{p} \operatorname{Tr}_{\mathcal{E}} \ln \left[1+f_{2}\left(1-f_{1}\right) T_{p}\left(e^{i \chi}-1\right)\right. \\
& \left.+\left(1-f_{2}\right) f_{1} T_{p}\left(e^{-i \chi}-1\right)\right] .
\end{aligned}
$$

Here we used the matrix identity $\ln \operatorname{det}(\mathbf{M})=\operatorname{Tr} \ln (\mathbf{M})$ and the trace $\operatorname{Tr}_{\mathcal{E}}$ is taken in energy indices. The logarithm is taken assuming convolution over internal energy indices, e.g., $\left(f_{1} f_{2}\right)_{\mathcal{E}^{\prime} \mathcal{E}^{\prime \prime}}=(2 \pi)^{-1} \int d \mathcal{E}_{1} f_{1}\left(\mathcal{E}^{\prime}, \mathcal{E}_{1}\right) f_{2}\left(\mathcal{E}_{1}, \mathcal{E}^{\prime \prime}\right)$.

In the following we show that the same result is obtained within the circuit theory of mesoscopic transport. ${ }^{24-26}$ In this case the system is represented by discrete circuit elements as depicted in Fig. 2. The cumulant generating function of the transferred charge is given by ${ }^{16,22}$

$$
\mathcal{S}(\chi)=\sum_{p} \operatorname{Tr} \ln \left[\check{1}+\frac{T_{p}}{2}\left(\frac{\left\{\check{G}_{1}(\chi), \check{G}_{2}(0)\right\}}{2}-\check{1}\right)\right],
$$

where

$$
\check{G}_{i}(0)=\left(\begin{array}{cc}
1 & 2 h_{i} \\
0 & -1
\end{array}\right)
$$

are the quasiclassical Keldysh-Green's functions of the terminals with $h_{i}=1-2 f_{i}$. The counting field is incorporated 
through the gauge transformation of the Keldysh-Green's function of the left terminal,

$$
\check{G}_{1}(\chi)=e^{-i \chi \check{\tau}_{1} / 2} \check{G}_{1}(0) e^{i \chi \check{\tau}_{1} / 2},
$$

where

$$
\check{\tau}_{1}=\left(\begin{array}{ll}
0 & 1 \\
1 & 0
\end{array}\right)
$$

in Keldysh space. The logarithm and the trace in Eq. (6) are taken assuming the convolution both in Keldysh and in energy indices.

Equation (6) can be simplified by using matrix representation in Keldysh space. After rewriting $\operatorname{Tr} \ln (\cdots)=\ln \operatorname{det}(\cdots)$ and taking the determinant by blocks using Eq. (A1), the result coincides with Eq. (5). This proves the equivalence of the circuit-theory expression for $\mathcal{S}(\chi)[\mathrm{Eq} .(6)]$ and the Levitov determinant formula [Eq. (1)] for a coherent twoterminal scatterer. The circuit-theory approach becomes advantageous in the case of several scatterers in series ${ }^{23,54}$ or for a multiterminal mesoscopic conductor with large number of conduction channels. ${ }^{27}$ It gives the charge-transfer statistics in terms of the scattering properties of individual elements, thus circumventing a nontrivial task of obtaining the scattering matrix of the composite system and averaging over the phase shifts.

Equation (5) is valid for a dc bias applied and energydependent transmission probabilities. In this case the occupation numbers $f_{1,2}$ are diagonal in energy and commute with each other and with $T_{p}(\mathcal{E})$. The trace over energy reduces simply to the integration and we obtain

$$
\begin{aligned}
\mathcal{S}(\chi)= & \frac{t_{0}}{\pi} \sum_{p} \int d \mathcal{E} \ln \left\{1+\left[1-f_{1}(\mathcal{E})\right] f_{2}(\mathcal{E}) T_{p}\left(e^{i \chi}-1\right)\right. \\
& \left.+f_{1}(\mathcal{E})\left[1-f_{2}(\mathcal{E})\right] T_{p}\left(e^{-i \chi}-1\right)\right\} .
\end{aligned}
$$

Here $t_{0}$ is the measurement time which is the largest time scale in the problem, much larger than the characteristic time scale on which the current fluctuations are correlated. ${ }^{55}$ The form of $\mathcal{S}(\chi)$ reveals that the elementary charge-transfer processes are single-electron transfers to the left and to the right. The electron transfers at different energies and in different channels are independent. The term $\left[1-f_{1}(\mathcal{E})\right] f_{2}(\mathcal{E}) T_{p} e^{i \chi}$ in Eq. (10) describes the electron transfer from the right to the left lead at energy $\mathcal{E}$ in the channel $p$. The probability of this process is proportional to the probability $f_{2}(\mathcal{E})$ that the state in the right lead is occupied, the probability $1-f_{1}(\mathcal{E})$ that the state in the left lead is empty, and the probability $T_{p}(\mathcal{E})$ of transfer across the scatterer. A similar analysis holds for the electron transfer from left to right. The left and right transfers are correlated because $\mathcal{S}(\chi)$ is not a sum of the left- and right-transfer generating functions.

Equation (5) is also valid for the time-dependent voltage applied and energy-independent transmission probabilities. In this case $f_{1,2}\left(\mathcal{E}^{\prime}, \mathcal{E}^{\prime \prime}\right)$ do not commute with each other. [However, the order of $f_{1}$ and $f_{2}$ can be exchanged as shown by Eqs. (5a) and (5b).] The logarithm has to be calculated with the matrix structure of $f_{1,2}$ in energy indices taken into account because time-dependent drive mixes the electron states with different energies.

A few remarks on applicability of our approach are in order. The circuit theory we have used applies to instantaneous scattering at the junction, with the frequency $\omega$ of the bias voltage much smaller than the inverse dwell time $\tau_{d}^{-1}$. It also applies for a dot sandwiched between two terminals and $\omega$ much smaller than the inverse $R C$ time $\tau_{R C}^{-1}$ of the charge relaxation on the dot. In a typical experimental situation $\tau_{R C} \ll \tau_{d},{ }^{56,57}$ with $\omega$ limited by the dwell time $\omega \ll \tau_{d}^{-1}$. However, as shown in Ref. 39, the photon-assisted noise $S_{I}(\omega)$ in the leading order $\left(e V_{0} / \omega\right)^{2} \ll 1$ in driving amplitude $V_{0}$ does not depend on $\tau_{d}$ : in a weakly driven junction the frequency range is set by the $R C$ time, $\omega \ll \tau_{R C}^{-1}$. The dwell time becomes important for larger voltage amplitudes. For $e V_{0} / \omega$ $\gtrsim 1$ a maximum in $\partial S_{I} / \partial \omega$ develops at the frequency $\omega$ $\sim \tau_{d}^{-1}$ because the photon-assisted noise $S_{I}(\omega)$ probes the electronic distribution function $f(\mathcal{E} ; t)=\int d \tau f(t+\tau / 2, t$ $-\tau / 2) e^{i \mathcal{E} \tau}$ which relaxes on a time scale given by $\tau_{d} \cdot{ }^{56,57} \mathrm{At}$ frequencies $\omega \sim \tau_{d}^{-1}$, the differential noise $\partial S_{I} / \partial V_{0}$ as a function of $V_{0}$ is increased with respect to the case $\omega \ll \tau_{d}^{-1}$. The increase is of the order of $20 \%$ with the distances between maxima and minima only weakly affected. This is consistent with the experiments of Schoelkopf et al. ${ }^{36}$ and Kozhevnikov et $a l .{ }^{37}$ which can be described by assuming the instantaneous scattering even though the frequency of the ac signal applied is comparable or larger than the inverse dwell time.

\section{ELEMENTARY CHARGE-TRANSFER PROCESSES AT $T_{e}=\mathbf{0}$}

\section{A. Decomposition of the cumulant generating function into elementary processes}

In this section we study elementary charge-transfer processes in a two-terminal junction with constant transmission eigenvalues $\left\{T_{p}\right\}$ and time-dependent voltage $V(t)$ applied. The cumulant generating function $\mathcal{S}(\chi)$ is given by Eq. (5). To identify the elementary processes we diagonalize the operator under the logarithm in energy indices. As a first step, we rewrite Eq. (5b) in terms of $\widetilde{h} \equiv h_{1}$ and $h \equiv h_{2}$,

$$
\begin{aligned}
\mathcal{S}(\chi)= & 2 \sum_{p} \operatorname{Tr}_{\mathcal{E}} \ln \left[1-T_{p}(1-h \tilde{h}) \sin ^{2}(\chi / 2)\right. \\
& \left.-i T_{p}(h-\tilde{h}) \sin (\chi / 2) \cos (\chi / 2)\right],
\end{aligned}
$$

where $h\left(\mathcal{E}^{\prime}, \mathcal{E}^{\prime \prime}\right)=\tanh \left(\mathcal{E}^{\prime} / 2 T_{e}\right) 2 \pi \delta\left(\mathcal{E}^{\prime}-\mathcal{E}^{\prime \prime}\right)$ and $\tilde{h}=U h U^{\dagger} .^{58}$ The $\mathcal{S}(\chi)$ further simplifies in the zero-temperature limit, in which the Hermitian $h$ operators are involutive $h^{2}=\widetilde{h}^{2}=1$ and satisfy $[h,\{h, \widetilde{h}\}]=[\tilde{h},\{h, \widetilde{h}\}]=0$. Thus the eigensubspaces of $\{h, \widetilde{h}\}$ are invariant with respect to $h, \tilde{h}$, and $h \tilde{h}$, and the diagonalization problem reduces to the subspaces of lower dimension. The typical eigensubspaces of $\{h, \widetilde{h}\}$ are twodimensional and spanned by the eigenvectors $\mathbf{v}_{\alpha}$ and $\mathbf{v}_{-\alpha}$ $=h \mathbf{v}_{\alpha}$ of $h \tilde{h}$ which correspond to the eigenvalues $e^{ \pm i \alpha}$ ( $\alpha$ is real). The diagonalization procedure in invariant subspaces is described in detail in Ref. 49.

For computational reasons it is convenient to impose periodic boundary conditions on the voltage drive $V(t+\tau)$ 
$=V(t)$ with the period $\tau=2 \pi / \omega$. In this case the operator $\tilde{h}$ $=U h U^{\dagger}$ couples only energies which differ by an integer multiple of $\omega$. This allows us to map the energy indices into the interval $0<\mathcal{E}<\omega$ while retaining the discrete matrix structure in steps of $\omega$. The operator $h \widetilde{h}$ in energy representation is given by

$$
\begin{aligned}
(h \widetilde{h})_{n m}(\mathcal{E}) & \equiv(h \widetilde{h})(\mathcal{E}+n \omega, \mathcal{E}+m \omega) \\
& =\operatorname{sgn}(\mathcal{E}+n \omega) \sum_{k=-\infty}^{\infty} a_{n+k} a_{m+k}^{*} \operatorname{sgn}(\mathcal{E}-k \omega-e \bar{V}),
\end{aligned}
$$

with

$$
a_{n}=\frac{1}{\tau} \int_{0}^{\tau} d t \exp \left[-i \int_{0}^{t} d t^{\prime} e \Delta V\left(t^{\prime}\right)\right] e^{i n \omega t} .
$$

Here $\bar{V}=(1 / \tau) \int V(t) d t$ is the dc voltage offset and $\Delta V(t)$ $=V(t)-\bar{V}$ is the ac voltage component. The coefficients $a_{n}$ satisfy

$$
\sum_{k=-\infty}^{\infty} a_{n+k} a_{m+k}^{*}=\delta_{n m}, \quad \sum_{n=-\infty}^{\infty} n\left|a_{n}\right|^{2}=0 .
$$

The cumulant generating function $\mathcal{S}(\chi)$ at zero temperature is given by $\mathcal{S}=\mathcal{S}_{1 L}+\mathcal{S}_{1 R}+\mathcal{S}_{2}$ with $^{49}$

$$
\mathcal{S}_{1 L, R}(\chi)=M_{L, R} \sum_{n} \sum_{k} \ln \left[1+T_{n} R_{n} p_{k L, R}\left(e^{i \chi}+e^{-i \chi}-2\right)\right]
$$

and

$$
\mathcal{S}_{2}(\chi)=\frac{t_{0}|e \bar{V}|}{\pi} \sum_{n} \ln \left[1+T_{n}\left(e^{-i \kappa \chi}-1\right)\right] .
$$

Here $M_{L}=t_{0} \omega_{1} / \pi, M_{R}=t_{0}\left(\omega-\omega_{1}\right) / \pi$, and $\omega_{1}=e \bar{V}-\lfloor e \bar{V} / \omega\rfloor \omega$, where $\lfloor x\rfloor$ is the largest integer less than or equal $x$. The coefficient $\kappa= \pm 1$ in Eq. (16) is related to direction of the charge transfer, with $\kappa=1 \quad(\kappa=-1)$ for $e \bar{V}>0(e \bar{V}<0)$. The total measurement time $t_{0}$ is much larger than the period $\tau$ and the characteristic time scale on which the current fluctuations are correlated.

The parameters $p_{k L, R}$ in Eq. (15) depend on the details of the time-dependent voltage drive. They are given by $p_{k L, R}$ $=\sin ^{2}\left(\alpha_{k L, R} / 2\right)$, where $e^{ \pm i \alpha_{k L(R)}}$ are the eigenvalues of $(h \widetilde{h})_{n m}$ calculated for $\mathcal{E} \in\left(0, \omega_{1}\right)\left[\mathcal{E} \in\left(\omega_{1}, \omega\right)\right]$. The eigenvalues are obtained using a finite-dimensional matrix $(h \widetilde{h})_{n m}$, with the cutoff in indices $n$ and $m$ being much larger than the characteristic scale on which $\left|a_{n}\right|$ vanish.

Equations (15) and (16) give the charge-transfer statistics in a two-terminal junction driven by a time-dependent voltage. The result is valid in the zero-temperature limit, with no thermal excitations present. The probability distribution of the number of charges $N$ transferred within measurement time is given by $\mathcal{P}(N)=(2 \pi)^{-1} \int_{-\pi}^{\pi} d \chi \exp [\mathcal{S}(\chi)-i N \chi]$. The cumulants of $N$ are given by $\mathcal{C}_{n}=\left[\partial_{i \chi}^{n} \mathcal{S}(\chi)\right]_{\chi=0}$ and are related to the higher-order current correlators at zero frequency. ${ }^{17,18}$

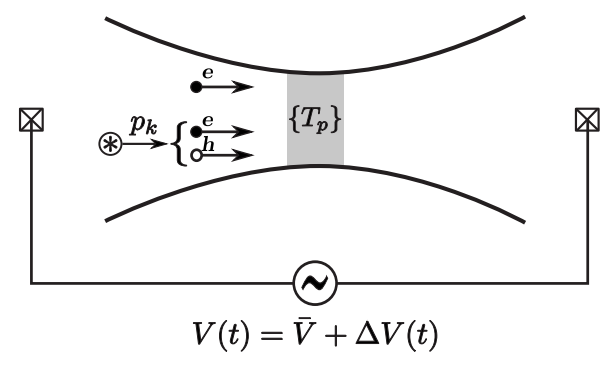

FIG. 3. The elementary charge-transfer processes in a voltagedriven junction. The unidirectional processes represent single electrons injected toward the contact due to excess dc offset voltage $\bar{V}$ applied. The bidirectional processes represent electron-hole pairs created by time-dependent voltage drive and injected toward the contact. The probabilities $p_{k}$ of pair creations depend on the ac voltage component $\Delta V(t)$.

For example, the average current, the current noise power, and the third cumulant of current fluctuations are given by $I=\left.\left(e / t_{0}\right) \partial_{i \chi} \mathcal{S}\right|_{\chi=0}, S_{I}=\left.\left(e^{2} / t_{0}\right) \partial_{i \chi}^{2} \mathcal{S}\right|_{\chi=0}$, and $C_{I}=\left.\left(e^{3} / t_{0}\right) \partial_{i \chi}^{3} \mathcal{S}\right|_{\chi=0}$, respectively. ${ }^{5}$

The elementary charge-transfer processes can be identified from the form of $\mathcal{S}$, similarly as in Refs. 60 and 61. The result is depicted schematically in Fig. 3. The $\mathcal{S}_{2}(\chi)$ describes unidirectional single-electron transfers due to the excess dc bias voltage $\bar{V}$ applied. The number of attempts for an electron to traverse the junction within measurement time $t_{0}$ is given by $t_{0}|e \bar{V}| / \pi$ (we take into account both spin orientations). The transfer events in different channels are independent. The term $T_{n} e^{-i \kappa \chi}$ in Eq. (16) describes a singleelectron transfer with probability $T_{n}$ in $n$th channel. The unidirectional charge-transfer processes give contributions to the average current and higher-order cumulants.

The $\mathcal{S}_{1 L}(\chi)$ and $\mathcal{S}_{1 R}(\chi)$ describe bidirectional chargetransfer processes. Different bidirectional processes are labeled by $k$ in Eq. (15). These processes represent electronhole pairs created in the source terminal by the timedependent voltage drive and injected toward the scatterer. The probability of such an electron-hole pair creation is given by $p_{k}$ and depends on the details of the ac voltage component $\Delta V(t)$. The charge transfer in $n$th channel occurs if one particle (e.g., electron) is transmitted and the hole is reflected or vice versa. The probability for the whole process is given by $T_{n} R_{n} p_{k}$. The bidirectional processes contribute to the noise and higher-order even cumulants. However, they give no contributions to the average current and higher-order odd cumulants because electrons and holes are transmitted with the same probability.

The two types of bidirectional processes $\mathcal{S}_{1 L}$ and $\mathcal{S}_{1 R}$ differ in the number of attempts $M_{L, R}$ and have different probabilities $p_{k L, R}$. The $M_{L, R}$ depend on the number $e \bar{V} / \omega$ of unidirectional attempts per period per spin. The simplest statistics is obtained for an integer value of $e \bar{V} / \omega$ for which $\mathcal{S}_{1 L}$ vanishes, in agreement with Ref. 7 .

\section{B. Comparison of different time-dependent voltages}

The elementary processes at zero temperature can be probed by noise measurements. In what follows we compare 


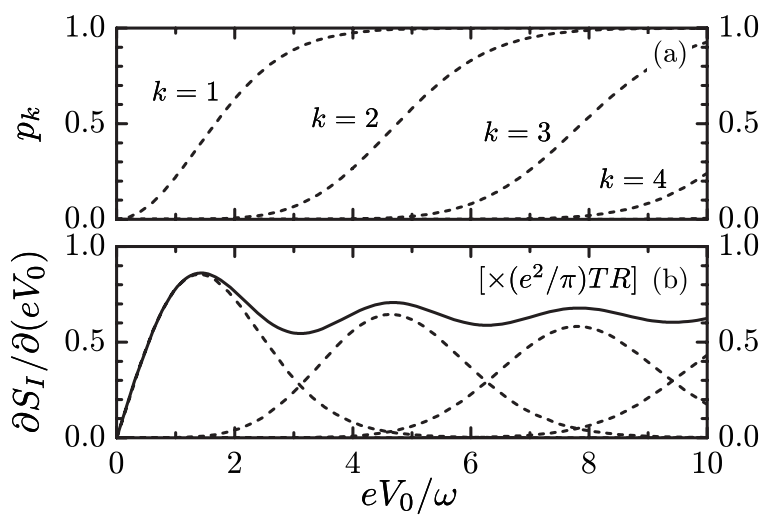

FIG. 4. The probabilities $p_{k}$ of electron-hole pair creations for harmonic drive $V(t)=V_{0} \cos (\omega t)$ as a function of the amplitude $V_{0}$ [panel (a)]. With increasing $V_{0}$ more and more pairs are created per voltage cycle. The decomposition of the differential noise $\partial S_{I} / \partial\left(e V_{0}\right)$ (solid line) into contributions of elementary processes (dashed lines) is shown in panel (b).

the elementary processes and the noise generated by different time-dependent voltages. We focus on standard periodic voltage signals such as cosine, square, triangle, and sawtooth. We also present results for Lorentzian voltage pulses which provide the simplest charge-transfer statistics for certain amplitudes. The applied voltage is characterized by coefficients $a_{n}$ given by Eq. (13). These coefficients are calculated explicitly in Appendix, Sec. B for the driving voltages of interest.

We first consider ac voltage drive with no dc bias applied, $\bar{V}=0$. In this case only the bidirectional processes of $R$-type remain, $\mathcal{S}(\chi)=\mathcal{S}_{1 R}(\chi)$. The number of attempts during the measurement time is given by $M=2 t_{0} / \tau$ which corresponds to a single attempt per voltage cycle per spin. The current noise power is given by

$$
S_{I}=\frac{2 e^{2} \omega}{\pi}\left(\sum_{p} T_{p} R_{p}\right)\left(\sum_{k} p_{k}\right)
$$

with the probabilities $p_{k}$ of electron-hole pair creations obtained from the eigenvalues of $(h \tilde{h})_{n m}$, as discussed in Sec. III A.

The probabilities $p_{k}$ for the harmonic voltage drive $V(t)$ $=V_{0} \cos (\omega t)$ are shown in Fig. 4(a). As the amplitude $V_{0}$ increases the probabilities $p_{k}$ also increase and new pairs start to enter the transport. This results in the oscillatory change in the slope of $S_{I}$ as a function of $V_{0}$. The decomposition of the differential noise $\partial S_{I} / \partial V_{0}$ into elementary processes is shown in Fig. 4(b). The differential noise $\partial S_{I} / \partial V_{0}$ for different time-dependent voltages is shown in Fig. 5 for comparison.

The bidirectional processes with the unit probability $p_{k}$ $=1$ represent electron-hole pairs which are created and injected toward the scatterer in each voltage cycle. In this case the electron and hole transfers are statistically independent. This can be seen from the corresponding cumulant generating function which reduces to $\mathcal{S}_{n k}=\mathcal{S}_{n k}^{e}+\mathcal{S}_{n k}^{h}$, where $\mathcal{S}_{n k}^{e, h}(\chi)$ $=\left(2 t_{0} / \tau\right) \ln \left[1+T_{n}\left(e^{\mp i \chi}-1\right)\right]$ describe electron and hole trans-

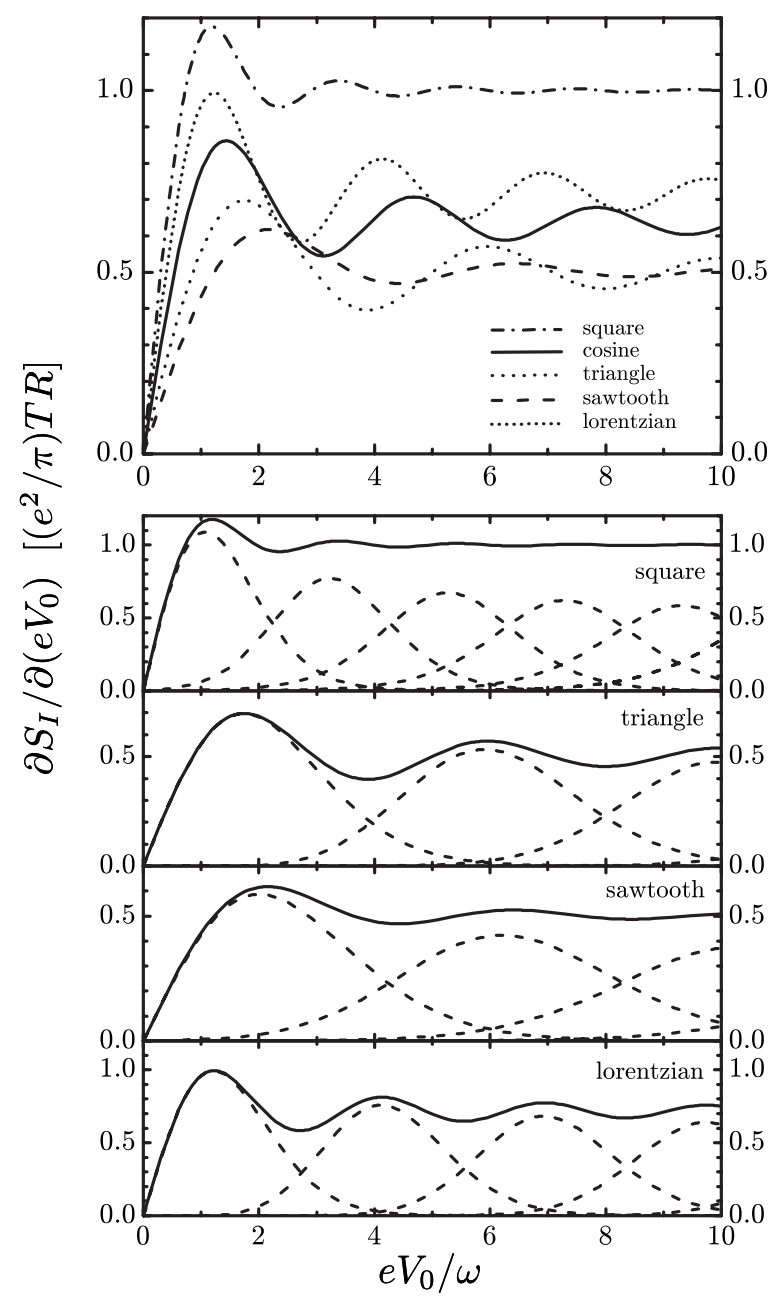

FIG. 5. The differential noise $\partial S_{I} / \partial\left(e V_{0}\right)$ as a function of the amplitude $V_{0}$ for different ac driving voltages $(\bar{V}=0)$ is shown in top panel: square (dashed-dotted), cosine (solid), triangle (dotted), sawtooth (dashed), and Lorentzian pulses (short-dotted line). The width of Lorentzian pulses is $\tau_{L}=0.1 \tau$. The oscillations are due to elementary processes which are created as the voltage amplitude increases (cf. Fig. 4). The decomposition of the differential noise into contributions of elementary processes is shown in lower panels.

port. The electron-hole pairs which are created with the probability $0<p_{k}<1$ result in correlated electron and hole transfers. The corresponding cumulant generating function is given by $\mathcal{S}_{n k}(\chi)=\left(2 t_{0} / \tau\right) \ln \left[1+T_{n} R_{n} p_{k}\left(e^{i \chi}+e^{-i \chi}-2\right)\right]$ and cannot be partitioned into independent electron and hole contributions.

The interpretation of the shot noise in terms of electronhole pair excitations has been studied previously in Ref. 38 in the regime of low-amplitude harmonic driving $e V_{0} / \omega$ $\ll 1$. In this case only one electron-hole pair is excited per period with probability $p_{1} \approx\left(e V_{0} / 2 \omega\right)^{2} \ll 1$. Remarkably, Fig. 4 shows that the single-electron-hole pair is excited not only for small amplitudes but also for amplitudes comparable or even larger than the drive frequency. This extended range of validity can be covered by taking into account the higherorder terms in the expression for probability $p_{1}$ $\approx \sum_{n=1}^{\infty} n\left[J_{n}\left(e V_{0} / \omega\right)\right]^{2}$, where $J_{n}(x)$ denote Bessel functions (cf. Sec. IV). The first three terms approximate the exact $p_{1}$ 
shown in Fig. 4 to accuracy better than $0.3 \%$ for $e V_{0} / \omega \lesssim 2$.

For large driving amplitudes the charge-transfer statistics does not depend on the details of time-dependent drive 8,42 and can be characterized by an effective voltage $V_{\text {eff }}$ $=\tau^{-1} \int_{\tau} d t|V(t)|$ (here we assume $\bar{V}=0$ ). In this case there are $N=\left|e V_{\text {eff }}\right| / 2 \omega \gg 1$ processes with $p_{k}=1$. The charge-transfer statistics in the leading order in $N$ consists of $N_{e, h}=2 N t_{0} / \tau$ uncorrelated electrons and holes injected toward the contact in each transport channel during measurement time: $\mathcal{S}(\chi)$ $=N_{e} \sum_{n} \ln \left[1+T_{n}\left(e^{-i \chi}-1\right)\right]+N_{h} \sum_{n} \ln \left[1+T_{n}\left(e^{i \chi}-1\right)\right]$. This result can be interpreted by comparison with the generating function of a dc bias given by Eq. (16). The electrons (holes) are injected during time intervals $\tau_{e}\left(\tau_{h}=\tau-\tau_{e}\right)$ per voltage cycle in which $e V(t)>0[e V(t)<0]$. For a large number of injected particles, the time-dependent drive in these intervals can be replaced by effective dc voltages $V_{\mathrm{eff}}^{(e)}$ and $V_{\mathrm{eff}}^{(h)}$. The number of attempts is given by $N_{i}=\left(t_{0} / \pi\right)\left(\tau_{i} / \tau\right)\left|e V_{\mathrm{eff}}^{(i)}\right|(i=e, h)$, in agreement with Eq. (16). The noise generated is the sum of independent electron and hole contributions $S_{I}$ $=\left(\mid e^{3} V_{\text {eff }} / / \pi\right) \sum_{n} T_{n} R_{n}$. This explains the asymptotic behavior of differential noise at large driving amplitudes shown in Figs. 4 and 5.

In the following we consider time-dependent voltage drive with a nonzero dc offset $\bar{V} \neq 0$ which creates both unidirectional and bidirectional elementary processes. The unidirectional processes generate dc noise which is given by $S_{\mathrm{dc}}=\left(e^{2} / \pi\right)\left(\Sigma_{p} T_{p} R_{p}\right) e \bar{V}$. The bidirectional processes given by Eq. (15) generate the excess noise $S_{I}-S_{\mathrm{dc}}$ $=\left.\left(e^{2} / t_{0}\right) \partial_{i \chi}^{2} \mathcal{S}_{1}\right|_{\chi=0}$,

$$
S_{I}-S_{\mathrm{dc}}=\frac{2 e^{2} \omega}{\pi}\left(\sum_{p} T_{p} R_{p}\right) \sum_{k}\left[\bar{v} p_{k L}+(1-\bar{v}) p_{k R}\right]
$$

where $\bar{v}=e \bar{V} / \omega-\lfloor e \bar{V} / \omega\rfloor$ is the fractional part of $e \bar{V} / \omega$. For $\bar{v} \neq 0,1$ there are two types of bidirectional processes (labeled by $L$ and $R$ ) with different numbers of attempts and different probabilities of electron-hole pair creations.

We study bidirectional processes first in the regime $V_{0}$ $=\bar{V}$ in which the ac amplitude and the dc offset increase simultaneously. The excess noise $S_{I}-S_{\mathrm{dc}}$ for different timedependent voltages is shown in Fig. 6(a) for comparison. The probabilities $p_{k L(R)}$ as a function of voltage are shown in Fig. 6(b) for the cosine voltage drive. The decomposition of the excess noise into contributions of elementary processes is shown in Fig. 6(c). For the cosine voltage drive there are two bidirectional processes (one of $L$ type and another of $R$ type) which are excited per period. The $L$-type processes transform continuously into the $R$-type ones at integer values of $e \bar{V} / \omega$, while the $R$-type processes disappear. The steplike evolution of the probabilities of $R$-type processes as a function of voltage does not introduce discontinuities in the current noise because the corresponding number of attempts vanishes. Instead, the interplay between $L$ and $R$ processes results in kinks and the local minima at integer values of $e \bar{V} / \omega$, as shown in Fig. 6(a). The $L$-type ( $R$-type) processes give the dominant contributions as $e \bar{V} / \omega$ approaches the integer val-
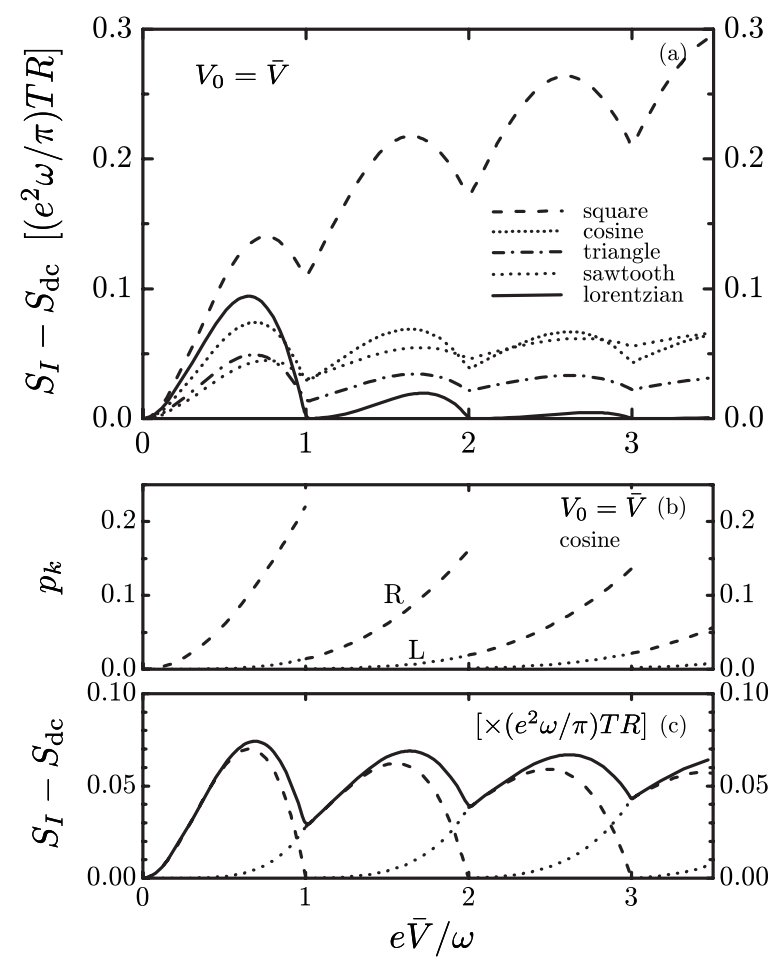

FIG. 6. The excess noise $S_{I}-S_{\mathrm{dc}}$ is shown in panel (a) for different time-dependent bias voltages: square (dashed), cosine (shortdotted), triangle (dashed-dotted), sawtooth (dotted), and Lorentzian pulses (solid line). The width of Lorentzian pulses is $\tau_{L}=0.1 \tau$. The amplitude and the dc offset increase simultaneously, $V_{0}=\bar{V}$. The probabilities $p_{k L}$ (dotted lines) and $p_{k R}$ (dashed lines) of bidirectional elementary processes are shown in panel (b) for the cosine voltage drive $V(t)=\bar{V}+\bar{V} \cos (\omega t)$. Only one elementary process of the $L$ type and one of the $R$ type can be created per period $(k=1)$. The decomposition of the excess noise $S_{I}-S_{\mathrm{dc}}$ into contributions of elementary processes is shown in panel (c).

ues from the left (right) because of the number of attempts which is proportional to $\bar{v}(1-\bar{v})$.

The Lorentzian voltage drive is special because it provides the simplest one-particle charge-transfer statistics. This is achieved for impulses carrying an integer number of charge quanta $e V_{0} / \omega=N$ at offset voltages $\bar{V} \geq V_{0}$. In this case the Lorentzian pulses do not create electron-hole pairs and only single-particle processes given by Eq. (16) remain. The charge-transfer statistics in each transport channel is $e x$ actly binomial. ${ }^{41,42}$ The noise is reduced to the minimal noise level $S_{\mathrm{dc}}$ of the effective dc bias, as shown in Fig. 6(a). A formal reason for this is the vanishing of the coefficients $a_{n}=0$ for $n<-N$ [cf. Eqs. (29) and (A9)]. A many-body state which is created by optimal Lorentzian pulses has been obtained by Keeling et al. ${ }^{43}$ recently.

Now we focus on regime in which the ac component $\Delta V(t)$ of the drive is fixed and the dc offset $\bar{V}$ changes. At low temperatures, the shot noise $S_{I}$ is a piecewise linear function of the de voltage offset $\bar{V}$ with kinks corresponding to integer multiples of the driving frequency $e \bar{V} / \omega=N$ and slopes which depend on the shape and the amplitude of the ac voltage component. ${ }^{33,34}$ The differential noise $\partial S_{I} / \partial(e \bar{V})$ 

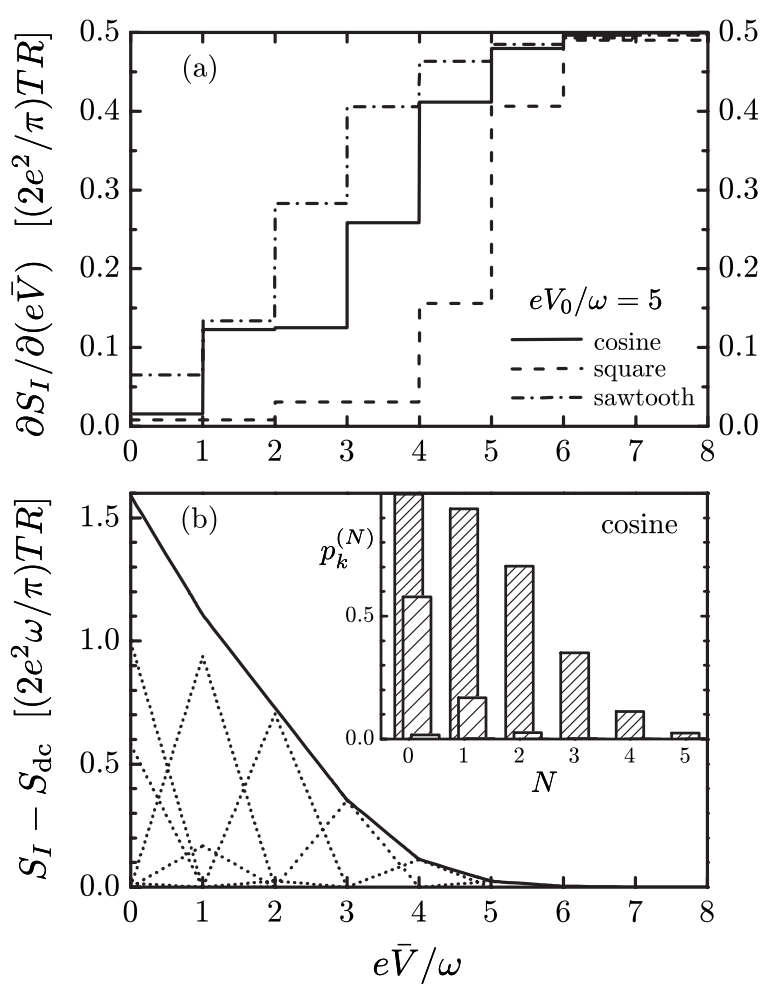

FIG. 7. The differential noise $\partial S_{I} / \partial(e \bar{V})$ as a function of $\mathrm{dc}$ offset $\bar{V}$ is shown in panel (a) for different time-dependent bias voltages: cosine (solid), square (dashed), and sawtooth (dasheddotted line). The ac amplitude of the drive is kept constant, $e V_{0} / \omega=5$. The excess noise $S_{I}-S_{\mathrm{dc}}$ as a function of $\bar{V}$ (solid line) and the decomposition into contributions of elementary processes (dotted lines) are shown for the cosine drive in panel (b) [cf. Eq. (18)]. The probabilities of elementary processes are shown in the inset.

consists of a series of steps, as shown in Fig. 7(a). The piecewise linear dependence of noise can be understood in terms of probabilities of elementary processes. ${ }^{7}$ For the ac component fixed, the probabilities $p_{k R}$ and $p_{k L}$ of electron-hole pair creations are piecewise constant as a function of $\bar{V}$ and can be relabeled by $p_{k R}(\bar{V}) \equiv p_{k}^{(N)}$ and $p_{k L}(\bar{V}) \equiv p_{k}^{(N+1)}$ where $N$ $=\lfloor e \bar{V} / \omega\rfloor$. This results in the piecewise linear dependence of the excess noise given by Eq. (18) as a function of $\bar{V}$. For the dc offset in the interval $m-1 \leq e \bar{V} / \omega<m$ with $m$ integer, the excess noise is a linear combination of processes $p_{k}^{(m-1)}$ and $p_{k}^{(m)}(k=1,2, \ldots)$ with the number of attempts proportional to $1-\bar{v}$ and $\bar{v}$, respectively. For $m \leq e \bar{V} / \omega<m+1$ a different set of processes $p_{k}^{(m)}$ and $p_{k}^{(m+1)}$ contributes and the excess noise changes slope. The excess noise for harmonic drive is shown in Fig. 7(b) (solid line) with decomposition into contributions of elementary processes (dotted lines). The probabilities of elementary processes are shown in the inset.

\section{Beam-splitter geometry}

Here we study a multiterminal beam-splitter geometry depicted in Fig. 8. The source terminal is biased with a time-

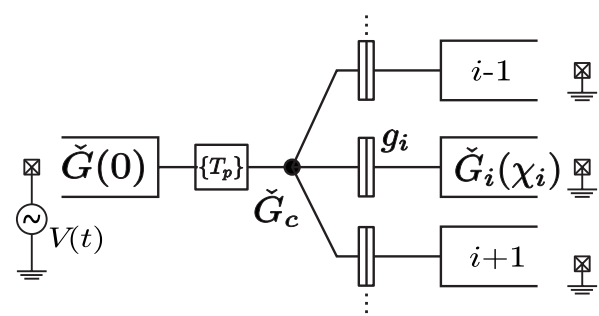

FIG. 8. Schematic representation of a multiterminal beam splitter. The source terminal is biased with a time-dependent voltage $V(t)$ with respect to the outgoing terminals. The total conductance $g_{\Sigma}=\Sigma_{i} g_{i}$ of the outgoing leads is assumed to be much larger than the conductance $g=\left(e^{2} / \pi\right) \Sigma_{p} T_{p}$ of the source contact.

dependent periodic voltage $V(t)$ and through a mesoscopic conductor attached to several outgoing terminals. The conductor is characterized by a set of transmission eigenvalues $\left\{T_{p}\right\}$ and the outgoing leads by conductances $g_{i}$. We are interested in the limit in which the outgoing leads play a role of a detector and only weakly perturb the charge transfer across the conductor. This is achieved for the conductance $g_{\Sigma} \equiv \Sigma_{i} g_{i}$ to the outgoing leads much larger than the conductance $g \equiv\left(e^{2} / \pi\right) \Sigma_{p} T_{p}$ of a conductor. In this case the particles which traverse the conductor enter the outgoing terminals with negligible back reflection into the source terminal. A similar setup with spin-selective outgoing contacts has been used in Ref. 61 to reveal singlet electron states.

The cumulant generating function is calculated using the circuit theory similarly as in Sec. II for the two-terminal case. In contrast to Sec. II, here we assign the counting fields $\chi_{i}$ to the outgoing terminals. Green's function $\check{G}(0)$ of the source terminal and Green's functions $\check{G}_{i}\left(\chi_{i}\right)$ of the outgoing ones are given by

$$
\check{G}(0)=\left(\begin{array}{cc}
1 & 2 \tilde{h} \\
0 & -1
\end{array}\right)
$$

and

$$
\check{G}_{i}\left(\chi_{i}\right)=e^{-i \chi_{i} \check{\tau}_{1} / 2}\left(\begin{array}{cc}
1 & 2 h \\
0 & -1
\end{array}\right) e^{i \chi_{i} \check{\tau}_{1} / 2} .
$$

Here $\tilde{h}$ and $h$ are the matrices in energy indices defined in Sec. III A. Green's function $\check{G}_{c}$ of the internal node is given by matrix current conservation and normalization condition $\check{G}_{c}^{2}=1$. In the limit $g_{\Sigma} \gg g$, the central node is strongly coupled to the outgoing terminals and the $\check{G}_{c}$ can be obtained in the lowest order with the terminal $\check{G}$ unattached. For simplicity we assume tunnel couplings to the outgoing terminals. In this case matrix current conservation reduces to ${ }^{22,24}$

$$
\left[\sum_{i} g_{i} \check{G}_{i}\left(\chi_{i}\right), \check{G}_{c}\right]=0 .
$$

In the following we work in the low-temperature limit in which $h^{2}=\widetilde{h}^{2}=1$. We seek for the solution in the form $\check{G}_{c}$ $=\mathbf{p}_{c} \cdot \check{\boldsymbol{\tau}}$ where $\check{\boldsymbol{\tau}}=\left(\check{\tau}_{1}, \check{\tau}_{2}, \check{\tau}_{3}\right)$ is the vector of Pauli matrices in Keldysh space. From Eqs. (19) and (20) we obtain $\mathbf{p}_{c}$ 
$=(h, i h c-s, i h s+c)$ where $c=\sum_{i} \widetilde{g}_{i} \cos \left(\chi_{i}\right), \quad s=\sum_{i} \widetilde{g}_{i} \sin \left(\chi_{i}\right)$, and $\tilde{g}_{i}=g_{i} / g_{\Sigma}$. The cumulant generating function of the charge transferred is given by

$$
\mathcal{S}(\{\chi\})=\sum_{p} \operatorname{Tr} \ln \left[\check{1}+\frac{T_{p}}{2}\left(\frac{\left\{\check{G}(0), \check{G}_{c}(\{\chi\})\right\}}{2}-\check{1}\right)\right],
$$

where summation over the internal Keldysh and energy indices is assumed. The diagonalization of $\left\{\check{G}, \check{G}_{c}\right\}_{\mathcal{E}^{\prime} \mathcal{E}^{\prime \prime}}$ is performed along the lines in Ref. 49.

For the cumulant generating function we obtain $\mathcal{S}(\{\chi\})$ $=\mathcal{S}_{1 L}+\mathcal{S}_{1 R}+\mathcal{S}_{2}$ where $\mathcal{S}_{1 L, R}$ are the contributions of bidirectional processes and $\mathcal{S}_{2}$ is the contribution of the unidirectional ones. The unidirectional processes are described by

$$
\mathcal{S}_{2}(\{\chi\})=\frac{t_{0} e \bar{V}}{\pi} \sum_{n} \ln \left(1+T_{n} \sum_{i} \widetilde{g}_{i}\left(e^{i \chi_{i}}-1\right)\right),
$$

where $t_{0}$ is the measurement time and $\bar{V}$ is the dc voltage offset (here we assume $e \bar{V}>0$ ). The bidirectional processes are described by

$$
\begin{aligned}
\mathcal{S}_{1 \alpha}(\{\chi\})= & M_{\alpha} \sum_{n} \sum_{k} \ln \left[1+p_{k \alpha} T_{n} R_{n}\left(\sum_{i} \widetilde{g}_{i}\left(e^{i \chi_{i}}+e^{-i \chi_{i}}-2\right)\right)\right. \\
& \left.+p_{k \alpha} T_{n}^{2}\left(\sum_{i<j} \tilde{g}_{i} \tilde{g}_{j}\left(e^{i \chi_{i}} e^{-i \chi_{j}}+e^{-i \chi_{i}} e^{i \chi_{j}}-2\right)\right)\right]
\end{aligned}
$$

Here $k$ labels the bidirectional processes, $n$ labels transport channels, $i$ and $j$ label the outgoing terminals, and $\alpha=L, R$. The number of attempts $M_{\alpha}$ and the probabilities $p_{k \alpha}$ are the same as in Sec. III A.

Equations (22) and (23) give the statistics of the charge transfer in a multiterminal beam splitter at low temperatures in the presence of a periodic time-dependent drive at the source terminal. The $\mathcal{S}_{1}(\{\chi\})$ and $\mathcal{S}_{2}(\{\chi\})$ have a direct physical interpretation. The unidirectional processes, which are described by $\mathcal{S}_{2}$, are the single-electron transfers across the structure due to the dc offset voltage $\bar{V}$ applied to the source terminal. The term $T_{n} \widetilde{g}_{i} e^{i \chi_{i}}$ in Eq. (22) represents the process in which an electron in the $n$th transport channel traverses the conductor with probability $T_{n}$ and enters the outgoing terminal $i$ with probability $\tilde{g}_{i}=g_{i} / g_{\Sigma}$.

On the other hand, the bidirectional processes represent the electron-hole pairs created in the source terminal and injected toward the conductor. The probabilities of such excitations are given by $p_{k \alpha}$. The interpretation of the cumulant generating function given by Eq. (23) can be obtained from a simple counting argument. The term $p_{k} T_{n} R_{n} \widetilde{g}_{i} e^{i \chi_{i}}$ in Eq. (23) represents the process in which electron-hole excitation is created, hole is reflected, and electron is transmitted into the outgoing terminal $i$. Similarly, the term $p_{k} T_{n} R_{n} \widetilde{g}_{i} e^{-i \chi_{i}}$ represents the process in which the electron is reflected and the hole is transmitted. Finally, the term $p_{k} T_{n}^{2} \tilde{g}_{i} \tilde{g}_{j} e^{i \chi_{i}} e^{-i \chi_{j}}(i \neq j)$ represents the process in which both particles are transmitted with electron entering terminal $i$ and hole entering terminal $j$.

Charge-transfer statistics and current correlations can be obtained using the cumulant generating function $\mathcal{S}(\{\chi\})$. For example, the current cross correlation between different terminals $i$ and $j$ is given by $S_{i j}=\left.\left(e^{2} / t_{0}\right) \partial_{i \chi_{i}, i \chi_{j}}^{2} \mathcal{S}\right|_{\chi=0}$,

$$
S_{i j}=-\frac{2 e^{2} \omega}{\pi}\left(\sum_{n} T_{n}^{2}\right) \widetilde{g}_{i} \widetilde{g}_{j} \sum_{k}\left[\bar{v} p_{k L}+(1-\bar{v}) p_{k R}\right] .
$$

We find that only bidirectional processes in which both particles are transferred (one into the terminal $i$ and another into the terminal $j$ ) give the contributions to the cross correlation $S_{i j}$. This has been obtained previously by Rychkov et al. ${ }^{38}$ in the special case of harmonic drive in the limit of small driving amplitudes $\left(\bar{V}=0\right.$ and $\left.e V_{0} / \omega \ll 1\right)$.

The cross correlation $S_{i j}$ depends on bidirectional processes and is proportional to the excess noise $S_{I}-S_{\mathrm{dc}}$ of a two-terminal junction [Eq. (18)]. In a two-terminal setup, the excess noise is just a small correction to $S_{\mathrm{dc}}$ generated by unidirectional processes for a bias voltage with $\bar{V} \simeq V_{0}$ (Fig. $6)$. The contribution of $S_{\mathrm{dc}}$ component can be reduced by measuring current cross correlations between different outgoing terminals in the beam-splitter geometry with negligible backscattering.

\section{CUMULANTS AT $T_{e} \neq 0$}

The full counting statistics and the corresponding elementary transport processes obtained in Sec. III are valid description in the low-temperature limit only, in which electron-hole pairs are created by the applied voltage and no thermally excited pairs exist. Formally, the diagonalization of the operator in Eq. (11) in energy indices, which is needed to deduce the elementary processes, is based on the involution property of $h$ operators: $h^{2}=\widetilde{h}^{2}=1$. This property no longer holds at finite temperatures which are comparable to the applied voltage. Nevertheless, the method we use enables the efficient and systematic analytic calculation of the higherorder cumulants at finite temperatures.

The cumulants can be obtained directly from Eq. (11) by expansion in the counting field to the certain order before taking the trace. The trace of a finite number of terms can be taken in the original basis in which $h$ and $\tilde{h}$ are defined. In the following we illustrate the approach by calculation of the average current, the current noise power, and the third cumulant at finite temperatures. From Eq. (11) we obtain

$$
\begin{gathered}
\left.\partial_{i \chi} \mathcal{S}\right|_{\chi=0}=\sum_{p} T_{p} \operatorname{Tr}_{\mathcal{E}}(\tilde{h}-h) \\
\left.\partial_{i \chi}^{2} \mathcal{S}\right|_{\chi=0}=\sum_{p}\left\{T_{p} \operatorname{Tr}_{\mathcal{E}}(1-h \tilde{h})-\left(T_{p}^{2} / 2\right) \operatorname{Tr}_{\mathcal{E}}\left[(h-\tilde{h})^{2}\right]\right\} \\
\left.\partial_{i \chi}^{3} \mathcal{S}\right|_{\chi=0}=\sum_{p}\left\{\left(T_{p}^{3} / 2\right) \operatorname{Tr}_{\mathcal{E}}\left(\widetilde{h}^{3}-h^{3}\right)+(3 / 2) T_{p}^{2}\left(1-T_{p}\right)\right. \\
\left.\quad \times \operatorname{Tr}_{\mathcal{E}}[h \tilde{h}(\tilde{h}-h)]+T_{p}\left[1-\left(3 T_{p} / 2\right)\right] \operatorname{Tr}_{\mathcal{E}}(\tilde{h}-h)\right\}
\end{gathered}
$$

In energy representation, the operators $h$ and $\tilde{h}$ are given by 


$$
\begin{gathered}
h\left(\mathcal{E}^{\prime}, \mathcal{E}^{\prime \prime}\right)=h\left(\mathcal{E}^{\prime}\right) 2 \pi \delta\left(\mathcal{E}^{\prime}-\mathcal{E}^{\prime \prime}\right) \\
=\sum_{k, m} a_{k} a_{k+m}^{*} h\left(\mathcal{E}^{\prime}\right) 2 \pi \delta\left(\mathcal{E}^{\prime \prime}-\mathcal{E}^{\prime}-m \omega\right), \\
\widetilde{h}\left(\mathcal{E}^{\prime}, \mathcal{E}^{\prime \prime}\right)=\sum_{k, m} a_{k} a_{k+m}^{*} h\left(\mathcal{E}^{\prime}-k \omega-e \bar{V}\right) 2 \pi \delta\left(\mathcal{E}^{\prime \prime}-\mathcal{E}^{\prime}-m \omega\right) .
\end{gathered}
$$

Here $h(\mathcal{E})=\tanh \left(\mathcal{E} / 2 T_{e}\right)$ and we used the properties of $\left\{a_{n}\right\}$ given by Eq. (14). After integration over energy in Eq. (25) we obtain the average current $I=\left(e^{2} / \pi\right)\left(\Sigma_{p} T_{p}\right) \bar{V}$. The current noise power and the third cumulant are given by

$$
\begin{aligned}
S_{I}= & \frac{e^{2}}{\pi}\left[2 T_{e} \sum_{p} T_{p}^{2}+\sum_{p} T_{p}\left(1-T_{p}\right)\right. \\
& \left.\times \sum_{n=-\infty}^{\infty}\left|a_{n}\right|^{2}(e \bar{V}+n \omega) \operatorname{coth}\left(\frac{e \bar{V}+n \omega}{2 T_{e}}\right)\right]
\end{aligned}
$$

and

$$
\begin{aligned}
C_{I}= & \frac{e^{3}}{\pi}\left\{e \bar{V} \sum_{p} T_{p}\left(1-T_{p}^{2}\right)+3 \sum_{p} T_{p}^{2}\left(1-T_{p}\right)\right. \\
& \times \sum_{n=-\infty}^{\infty}\left|a_{n}\right|^{2}\left[2 T_{e} \operatorname{coth}\left(\frac{e \bar{V}+n \omega}{2 T_{e}}\right)\right. \\
& \left.\left.-(e \bar{V}+n \omega) \operatorname{coth}^{2}\left(\frac{e \bar{V}+n \omega}{2 T_{e}}\right)\right]\right\},
\end{aligned}
$$

respectively. For a dc voltage bias $a_{n}=\delta_{n, 0}$ and the noise and the third cumulant reduce to $S_{I}=e F I$ and $C_{I}=e^{2} F_{3} I$ at zero temperature. Here $F=\left[\Sigma_{p} T_{p}\left(1-T_{p}\right)\right] /\left(\Sigma_{p} T_{p}\right)$ is the Fano factor and $F_{3}=\left[\Sigma_{p} T_{p}\left(1-T_{p}\right)\left(1-2 T_{p}\right)\right] /\left(\Sigma_{p} T_{p}\right)$.

The average current is linear in dc voltage offset, which is consistent with the initial assumption of energy-independent transmission eigenvalues and instant scattering at the contact. The result for the current noise power [Eq. (27)] describes the photon-assisted noise for arbitrary periodic voltage drive. The coefficients $a_{n}$ for harmonic drive $V(t)=\bar{V}$ $+V_{0} \cos (\omega t)$ are given by the Bessel functions, $a_{n}$ $=J_{n}\left(e V_{0} / \omega\right)$, and Eq. (27) reduces to the previous results obtained by Lesovik and Levitov ${ }^{33}$ and Pedersen and Büttiker $^{34}$ (see also Ref. 1). The accurate noise measurements at finite temperatures in the presence of the harmonic driving are performed in Ref. 35 . The results are in agreement with Eq. (27).

In the following we discuss the low- and high-temperature limits of $S_{I}$ and $C_{I}$. At high temperatures $T_{e} \gg|e \bar{V}|, n_{0} \omega$, with $n_{0} \omega$ being the characteristic energy scale on which $\left|a_{n_{0}}\right|$ vanish, the current noise power reduces to the thermal equilibrium value $S_{I}=2 T_{e} G$, which is just a manifestation of the fluctuation-dissipation theorem. The third cumulant is in this regime proportional to the average current, $C_{I}=e^{2} F I$. At high temperatures $S_{I}$ and $C_{I}$ carry no information on the details of the time-dependent voltage drive.
At low temperatures $T_{e} \ll|e \bar{V}|, n_{0} \omega$, the current noise power reduces to

$$
S_{I}=\frac{e^{2}}{\pi}\left(\sum_{p} T_{p} R_{p}\right) \sum_{n=-\infty}^{\infty}|e \bar{V}+n \omega|\left|a_{n}\right|^{2} .
$$

The differential noise $\partial S_{I} / \partial \bar{V}$ is a piecewise constant function of $\bar{V}$ with steps given by ${ }^{33}$

$$
\begin{aligned}
\left(\Delta \frac{\partial S_{I}}{\partial \bar{V}}\right)_{e \bar{V} / \omega=N} & =\left.\frac{\partial S_{I}}{\partial \bar{V}}\right|_{N+0}-\left.\frac{\partial S_{I}}{\partial \bar{V}}\right|_{N-0} \\
& =\frac{2 e^{3}}{\pi}\left(\sum_{p} T_{p} R_{p}\right)\left|a_{-N}\right|^{2} .
\end{aligned}
$$

All steps add up to $\left(2 e^{3} / \pi\right) \Sigma_{p} T_{p} R_{p}$ because of $\Sigma_{n}\left|a_{n}\right|^{2}=1$ [see Fig. 7(a)].62 The steps in $\partial S_{I} / \partial \bar{V}$ have been measured for harmonic drive in normal ${ }^{36}$ and normal-superconductor ${ }^{37}$ junctions. In the superconducting state they appear at integer values of $2 e \bar{V} / \omega$, which can be interpreted as a signature of the elementary charge transport processes in units of $e^{*}=2 e$. The effective charge is doubled in the superconducting state due to the Andreev processes. We point out that for a general voltage drive, certain steps at integer values of $e^{*} \bar{V} / \omega=N$ may vanish if the corresponding coefficient $a_{-N}=0$. For example, for a square-shaped drive with integer amplitude $e^{*} V_{0} / \omega=m$, the steps at $e^{*} \bar{V} / \omega=m+2 k(k \neq 0)$ vanish [cf. Eq. (A4) and Fig. 7(a)].

At low temperatures, the third cumulant reduces to $C_{I}$ $=e^{2} F_{3} I$. Unlike the current noise power, the third cumulant at low temperatures does not depend on the ac component of the voltage drive. This is because the bidirectional processes, created by the ac voltage component, do not contribute to odd-order cumulants at low temperatures [recall Eq. (15)].

We conclude this section by comparison of two formulas for the current noise power at zero temperature. For simplicity we take $\bar{V}=0$. Equation (29) for the current noise power reduces to

$$
S_{I}=\frac{2 e^{2} \omega}{\pi}\left(\sum_{p} T_{p} R_{p}\right)\left(\sum_{n=1}^{\infty} n\left|a_{n}\right|^{2}\right) .
$$

Here we used Eq. (14) to restrict the summation to the positive $n$ only. On the other hand, the current noise power is also given by Eq. (17). Regardless the similar form, the physical content of these two equations is very different. Both equations give the same result for $S_{I}$ as a consequence of the invariance of trace. However, Eq. (17) has been obtained by taking the trace of the operator in Eq. (11) in the basis in which it is diagonal. The cumulant generating function given by Eq. (15) is decomposed into contributions of elementary and statistically independent processes. The terms proportional to $p_{k}$ which appear in Eq. (17) are the contributions of these processes to the noise. Equation (31) has been obtained by taking the trace of the operator in Eq. (11) in a basis in which it is not diagonal. Although the end result for $S_{I}$ is the same, the individual terms proportional to 
$n\left|a_{n}\right|^{2}$ which appear in Eq. (31) have no direct physical interpretation.

In the limit of small-amplitude voltage drive, $e V_{0} / \omega \ll 1$, only one electron-hole pair is excited per period with probability $p_{1}$. Comparing Eqs. (17) and (31) we obtain that in this case $p_{1}=\sum_{n=1}^{\infty} n\left|a_{n}\right|^{2}$. In fact, as shown in Fig. 4 for harmonic drive, the assumption of small amplitudes can be relaxed to the amplitudes comparable or even larger than the drive frequency. The accuracy of this approximation depends on the actual voltage drive (cf. Figs. 4 and 5).

\section{CHARGE-TRANSFER PROCESSES AT $T_{e} \neq 0$}

Here we study the effect of finite temperature on elementary charge-transfer processes. For a periodic voltage drive, the cumulant generating function $\mathcal{S}(\chi)$ in Eq. (11) can be recast in the following form:

$$
\mathcal{S}(\chi)=\frac{t_{0}}{\pi} \sum_{p} \int_{0}^{\omega} d \mathcal{E} \ln \left(1+\sum_{n=-\infty}^{\infty} q_{n}\left(e^{i n \chi}-1\right)\right) .
$$

Here $q_{n} e^{i n \chi}$ describes the process in which $n$ charges are transmitted through the scatterer with probability $q_{n}$. The charge transfer can occur in either direction depending on the sign of $n$. To obtain the probabilities $q_{n}$ we diagonalize the operator under the logarithm in Eq. (11) numerically, multiply all the eigenvalues, and take the inverse Fourier transformation with respect to $\chi$. For simplicity we focus on ac voltage drive with no dc offset. For a given ac drive, the probabilities $q_{n}$ depend on energy, temperature, and transmission, $q_{n} \equiv q_{n}\left(\mathcal{E} / \omega, T_{e} / \omega ; T\right)$. Transport properties of the junction enter through summation over the transmission eigenchannels $\left\{T_{p}\right\}$.

Equation (32) allows us to study the crossover from acdriven fluctuations at zero temperature to thermal fluctuations at temperatures much larger than the voltage drive. The important difference between the two regimes is that ac drive mixes electron states of different energies in contrast to thermal fluctuations which are diagonal in energy. As we have seen, this is also reflected in the charge-transfer statistics. The statistics for an ac drive (at zero temperature) can be interpreted in terms of electron-hole pairs after mapping the problem into energy interval set by the driving frequency $\omega$. The mixing of energy states is taken into account by diagonalization of the remaining matrix structure in energy. On the other hand, the statistics in the thermal limit is composed of electron transfers (in either direction) which are independent at different energies. In the crossover regime there is still some mixing of different energy states present and we use the probabilities $q_{n}$ in Eq. (32) with energy mapped in the $\omega$ interval. These different physical situations are depicted schematically in Fig. 1.

The probabilities $q_{n}$ are shown in Fig. 9 for cosine voltage drive $V(t)=V_{0} \cos (\omega t)$ and a transport channel of transmission $T_{p}=0.5$. We first discuss thermal limit of probabilities $q_{n}$ shown by solid lines in Figs. 9(a) and 9(b). For $\omega \gg T_{e}$, the mapping into interval $\omega$ is irrelevant since it is larger than the energy scale of thermal fluctuations. In this case the probabilities reduce to $q_{ \pm 1}=f(1-f) T_{p}$ and $q_{n}=0$ otherwise, in
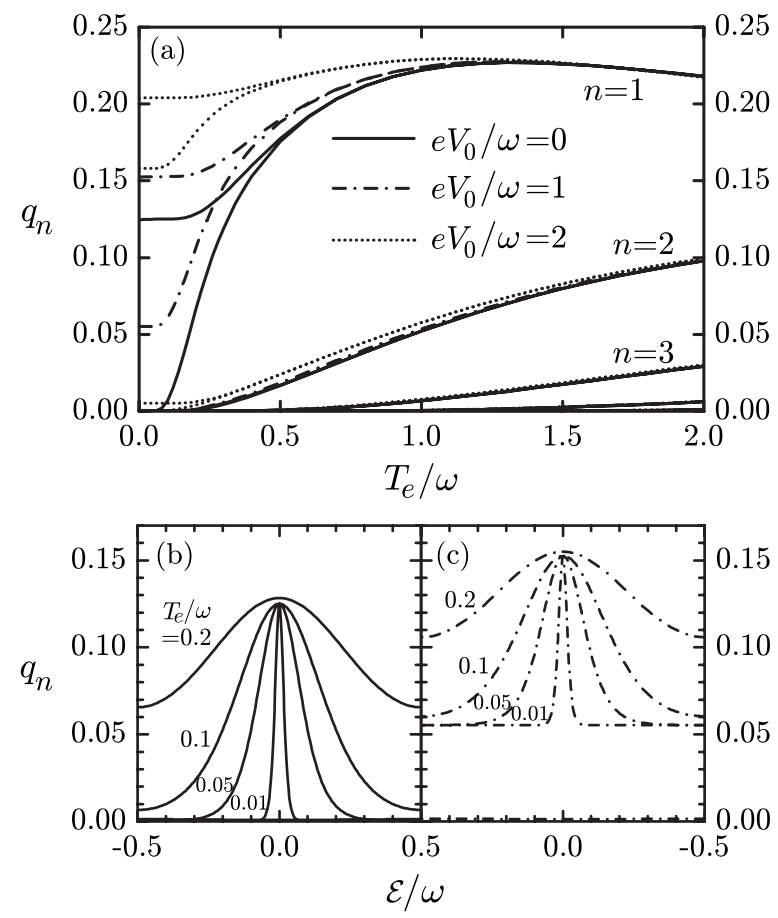

FIG. 9. The probabilities $q_{ \pm n}$ of multiple-charge transfers for harmonic voltage drive $V(t)=V_{0} \cos (\omega t)$ and transmission $T_{p}=0.5$. The temperature dependence of $q_{n}$ is shown in panel (a) for different driving amplitudes. The energy dependence of $q_{n}$ at low temperatures is indicated by plots for $\mathcal{E} / \omega=0$ (top line) and $\mathcal{E} / \omega=0.5$ (bottom line). The energy dependence of $q_{n}$ is shown in the thermal limit (b) $e V_{0} / \omega=0$ and for (c) $e V_{0} / \omega=1$. At zero temperature $q_{ \pm 1}$ do not depend on energy and are given by $q_{ \pm 1}=T(1$ $-T) p_{1}\left(e V_{0} / \omega\right)$ (cf. Fig. 4). As the temperature increases, multiplecharge transfers start to enter the transport. The limit of thermal fluctuations is reached at temperatures larger than the driving amplitude. It can be recast again into single-charge transfers independent in the full energy range.

accordance with Eq. (10) [cf. Fig. 9(b)]. As the mapping interval becomes comparable or smaller than the temperature, the higher-order "bands" $q_{n}(n= \pm 1, \pm 2, \ldots)$ start to appear. For $\omega \leqq 2 T_{e}$ the probabilities $q_{n}$ are independent of energy [Fig. 9(a)] and are given by

$$
q_{n}=\int_{-\pi}^{\pi} \frac{d \chi}{2 \pi} e^{\tilde{\mathcal{S}}(\chi)-i n \chi}
$$

with $\widetilde{\mathcal{S}}(\chi)=-4\left(T_{e} / \omega\right) \arcsin ^{2}\left[\sqrt{T_{p}} \sin (\chi / 2)\right]$. Here $\widetilde{\mathcal{S}}(\chi)$ is related to the cumulant generating function $\mathcal{S}(\chi)$ in thermal equilibrium by $\mathcal{S}(\chi)=\left(2 t_{0} / \tau\right) \Sigma_{p} \widetilde{\mathcal{S}}(\chi)$. The latter is obtained from Eq. (10) after energy integration. ${ }^{8}$

We emphasize again that the picture of multiple-charge transfers in thermal equilibrium limit is solely due to mapping into the energy interval $\omega$ in Eq. (32). Although such mapping is not needed because thermal fluctuations are diagonal in energy, we nevertheless perform it here to provide a limit for ac-driven processes as the temperature increases. At temperatures much larger than ac drive there is no mixing of different energy states and the statistics in the full energy 
range reduces to single-electron transfers which are independent at different energies [Eq. (10)].

Now we focus on the ac-driven processes and the crossover region between ac and thermal fluctuations as the temperature increases. For simplicity, we consider driving amplitudes $e V_{0} / \omega=1$ and $e V_{0} / \omega=2$. In this case $q_{ \pm 1}\left(T_{p}\right)$ $=T_{p}\left(1-T_{p}\right) p_{1}\left(e V_{0} / \omega\right)$ at zero temperature and do not depend on energy. These single-charge transfers originate from an electron-hole pair which is created with probability $p_{1}$ per voltage cycle (Fig. 4). As the temperature increases, the interplay of thermal and ac excitations introduces a nontrivial energy dependence of probabilities $q_{ \pm 1}$ shown in Fig. 9(c). It also modifies the dependence on transmission eigenvalues with $q_{ \pm 1}\left(T_{p}\right)$ no longer being proportional to $T_{p}\left(1-T_{p}\right)$ [cf. Eq. (33)]. As the temperature increases further, the multiplecharge transfers come into play as shown in Fig. 9(a). At temperatures larger than the driving amplitude the limit of thermal transport is reached with statistics which can be recast again in the form of single-charge transfers, independent at different energies.

\section{CONCLUSION}

We have studied charge-transfer statistics in a generic mesoscopic contact driven by a time-dependent voltage. We have obtained the analytic form of the cumulant generating function at zero temperature and identified the elementary charge-transfer processes. The unidirectional processes represent electrons which are injected from the source terminal due to excess dc bias voltage. The bidirectional processes represent electron-hole pairs which are created by the timedependent voltage bias and injected toward the contact. This interpretation is consistent with the charge-transfer statistics in a multiterminal beam-splitter geometry in which electrons and holes can be partitioned into different outgoing terminals.

The elementary charge-transfer processes can be probed by current noise power and higher-order current correlators. The bidirectional processes contribute to the noise and higher-order even cumulants of the transferred charge at low temperature and give no contributions to the average current and higher-order odd cumulants. For an ac voltage with no dc offset, the noise is entirely due to bidirectional processes. The individual processes can be identified from the oscillations of the differential noise $\partial S_{I} / \partial V_{0}$ as the amplitude $V_{0}$ of the drive increases.

A time-dependent voltage drive with a nonzero dc offset generates both unidirectional and bidirectional processes. The bidirectional processes give rise to the excess noise with respect to dc noise level which is set by the unidirectional processes. The excess noise can be probed by measuring current cross correlations between different outgoing terminals in the beam-splitter geometry. The cross correlations are only due to processes in which the incoming electron-hole pair is split and the particles enter different outgoing terminals.

The method we use enables the systematic calculation of the higher-order current correlators at finite temperatures by expansion of the cumulant generating function in the count- ing field. We have obtained the current noise power and the third cumulant for arbitrary periodic voltage applied.

We have also studied the effect of finite temperature on elementary charge-transfer processes. In the limits of low (high) temperatures the single-charge transfers occur in either direction due to ac excited (thermally excited) electronhole pairs. However, the nature of the two is very different: the ac drive mixes the electron states of different energies, while thermal fluctuations are diagonal in energy. In the crossover region there is still some mixing of different energy states present. Such an interplay of thermal and ac excitations can be interpreted in terms of multiple-charge transfers with probabilities dependent on energy and temperature.

\section{ACKNOWLEDGMENTS}

This work has been supported by the German Research Foundation (DFG) through Grants No. SFB 513 and No. SFB 767 and the Swiss National Science Foundation.

\section{APPENDIX}

\section{Determinants of block matrices}

Let $\mathbf{A}, \mathbf{B}, \mathbf{C}$, and $\mathbf{D}$ be the quadratic matrices of the same size. Then the following equalities hold:

$$
\operatorname{det}\left(\begin{array}{ll}
\mathbf{A} & \mathbf{B} \\
\mathbf{C} & \mathbf{D}
\end{array}\right)= \begin{cases}\operatorname{det}(\mathbf{A D}-\mathbf{B C}), & {[\mathbf{C}, \mathbf{D}]=0} \\
\operatorname{det}(\mathbf{D A}-\mathbf{B C}), & {[\mathbf{B}, \mathbf{D}]=0} \\
\operatorname{det}(\mathbf{D A}-\mathbf{C B}), & {[\mathbf{A}, \mathbf{B}]=0} \\
\operatorname{det}(\mathbf{A D}-\mathbf{C B}), & {[\mathbf{A}, \mathbf{C}]=0 .}\end{cases}
$$

In the case in which more than two blocks commute with each other, the corresponding determinants on the right-hand side of Eq. (A1) coincide.

\section{Coefficients $a_{n}$}

Here we calculate coefficients $\left\{a_{n}\right\}$ given by Eq. (13) for different time-dependent voltages. For the cosine voltage drive $V(t)=\bar{V}+V_{0} \cos (\omega t)$, the coefficients $a_{n}$ can be calculated using Jacobi-Anger expansion, ${ }^{63}$

$$
e^{i z \sin (\theta)}=\sum_{n=-\infty}^{\infty} J_{n}(z) e^{i n \theta},
$$

where $J_{n}$ are the Bessel functions of the first kind. From Eq. (13) we obtain

$$
a_{n}=J_{n}\left(e V_{0} / \omega\right)
$$

The square voltage drive is given by $\Delta V(t)=V_{0}$ for $0<t$ $<\tau / 2$ and $\Delta V(t)=-V_{0}$ for $\tau / 2<t<\tau$. For noninteger values of $e V_{0} / \omega$, the coefficients $a_{n}$ are given by

$$
a_{n}=\frac{2}{\pi} \frac{e V_{0}}{\omega} \frac{\sin \left[\left(n-e V_{0} / \omega\right) \pi / 2\right]}{n^{2}-\left(e V_{0} / \omega\right)^{2}} e^{i\left(n-e V_{0} / \omega\right) \pi / 2} .
$$

For integer values of $e V_{0} / \omega$, the coefficients $a_{n}$ are obtained by taking the limit in the previous formula. 
The sawtooth voltage drive is given by $\Delta V(t)=2 V_{0} t / \tau$ $-V_{0}$ for $0<t<\tau$. In this case

$$
\begin{aligned}
a_{n}= & \frac{1}{2 \sqrt{2 e V_{0} / \omega}} \exp \left(i \frac{\pi}{2} \frac{\left(e V_{0} / \omega+n\right)^{2}-e V_{0} / 2 \omega}{e V_{0} / \omega}\right) \\
& \times\left[\operatorname{erf}\left(\frac{\sqrt{\pi}\left(e V_{0} / \omega-n\right)}{\sqrt{2 e V_{0} / \omega}} e^{i \pi / 4}\right)\right. \\
& \left.+\operatorname{erf}\left(\frac{\sqrt{\pi}\left(e V_{0} / \omega+n\right)}{\sqrt{2 e V_{0} / \omega}} e^{i \pi / 4}\right)\right],
\end{aligned}
$$

where $\operatorname{erf}(z)=(2 / \sqrt{\pi}) \int_{0}^{z} d t \exp \left(-t^{2}\right)$ is the error function.

The triangle voltage drive is characterized by $\Delta V(t)$ $=4 V_{0} t / \tau-V_{0}$ for $0<t<\tau / 2$ and $\Delta V(t)=-4 V_{0} t / \tau+3 V_{0}$ for $\tau / 2<t<\tau$. In this case

$$
\begin{aligned}
a_{n}= & \frac{1}{2 \sqrt{e V_{0} / \omega}} \operatorname{Re}\left\{\exp \left(i \frac{\pi}{4} \frac{\left(e V_{0} / \omega+n\right)^{2}-e V_{0} / \omega}{e V_{0} / \omega}\right)\right. \\
& \times\left[\operatorname{erf}\left(\frac{\sqrt{\pi}\left(e V_{0} / \omega-n\right)}{2 \sqrt{e V_{0} / \omega}} e^{i \pi / 4}\right)\right. \\
& \left.\left.+\operatorname{erf}\left(\frac{\sqrt{\pi}\left(e V_{0} / \omega+n\right)}{2 \sqrt{e V_{0} / \omega}} e^{i \pi / 4}\right)\right]\right\} .
\end{aligned}
$$

The voltage drive which consists of Lorentzian voltage pulses of width $\tau_{L}$ is given by

$$
\begin{aligned}
\Delta V(t) & =-V_{0}+\frac{V_{0}}{\pi} \sum_{k=-\infty}^{\infty} \frac{\tau \tau_{L}}{(t-k \tau)^{2}+\tau_{L}^{2}} \\
& =-V_{0}+\frac{V_{0} \sinh \left(2 \pi \tau_{L} / \tau\right)}{\cosh \left(2 \pi \tau_{L} / \tau\right)-\cos (2 \pi t / \tau)} .
\end{aligned}
$$

The total voltage $V(t)=\bar{V}+\Delta V(t)$ varies between $V_{\min }=\bar{V}$ $+V_{0}\left[\tanh \left(\pi \tau_{L} / \tau\right)-1\right]$ and $V_{\max }=\bar{V}+V_{0}\left[\operatorname{coth}\left(\pi \tau_{L} / \tau\right)-1\right]$. The coefficients $a_{n}$ are given by

$$
a_{n}=e^{-i \pi e V_{0} / \omega} \int_{-1 / 2}^{1 / 2} d x \frac{\{\sin [\pi(x+i y)]\}^{e V_{0} / \omega}}{\{\sin [\pi(x-i y)]\}^{e V_{0} / \omega}} e^{i 2 \pi\left(e V_{0} / \omega+n\right) x},
$$

where $y=\tau_{L} / \tau$. For integer values $e V_{0} / \omega=N>0$ we obtain the simplified expressions,

$$
a_{n}=(-1)^{N} 2 \pi i \operatorname{Res}_{x=i y}\left(\frac{\sin ^{N}[\pi(x+i y)]}{\sin ^{N}[\pi(x-i y)]} e^{i 2 \pi(n+N) x}\right)
$$

for $n>-N, a_{n}=0$ for $n<-N$, and $a_{n=-N}=(-1)^{N} e^{-2 \pi N y}$. In particular, the coefficients $a_{n}$ for $e V_{0} / \omega=1$ are given by $a_{n}$ $=e^{-2 \pi n y}-e^{-2 \pi(n+2) y}$ for $n>-1, a_{-1}=-e^{-2 \pi y}$, and $a_{n}=0$ other wise. For $e V_{0} / \omega=2$ we obtain $a_{n}=e^{-2 \pi n y}\left(1-e^{-4 \pi y}\right)[n+1$ $\left.-(n+3) e^{-4 \pi y}\right]$ for $n>-2, a_{-2}=e^{-4 \pi y}$, and $a_{n}=0$ otherwise.
${ }^{1}$ Ya. M. Blanter and M. Büttiker, Phys. Rep. 336, 1 (2000).

${ }^{2}$ B. Reulet, J. Senzier, and D. E. Prober, Phys. Rev. Lett. 91, 196601 (2003).

${ }^{3}$ B. Reulet, in Nanophysics: Coherence and Transport, Lecture Notes of the Les Houches Summer School 2004 Vol. 81, edited by H. Bouchiat, Y. Gefen, S. Gueron, G. Montambaux, and J. Dalibard (Elsevier, Amsterdam, 2005).

${ }^{4}$ Yu. Bomze, G. Gershon, D. Shovkun, L. S. Levitov, and M. Reznikov, Phys. Rev. Lett. 95, 176601 (2005).

${ }^{5}$ L. S. Levitov and G. B. Lesovik, Pis'ma Zh. Eksp. Teor. Fiz. 58, 225 (1993) [JETP Lett. 58, 230 (1993)].

${ }^{6}$ L. S. Levitov and G. B. Lesovik, arXiv:cond-mat/9401004 (unpublished).

${ }^{7}$ D. A. Ivanov and L. S. Levitov, Pis'ma Zh. Eksp. Teor. Fiz. 58, 450 (1993) [JETP Lett. 58, 461 (1993)].

${ }^{8}$ L. S. Levitov, H.-W. Lee, and G. B. Lesovik, J. Math. Phys. 37, 4845 (1996).

${ }^{9}$ F. Hassler, M. V. Suslov, G. M. Graf, M. V. Lebedev, G. B. Lesovik, and G. Blatter, Phys. Rev. B 78, 165330 (2008).

${ }^{10}$ S. Pilgram, A. N. Jordan, E. V. Sukhorukov, and M. Büttiker, Phys. Rev. Lett. 90, 206801 (2003).

${ }^{11}$ A. N. Jordan, E. V. Sukhorukov, and S. Pilgram, J. Math. Phys. 45, 4386 (2004).

${ }^{12}$ Yu. V. Nazarov, Ann. Phys. 8, SI193 (1999).

${ }^{13}$ W. Belzig and Yu. V. Nazarov, Phys. Rev. Lett. 87, 197006 (2001).

${ }^{14}$ W. Belzig and Yu. V. Nazarov, Phys. Rev. Lett. 87, 067006
(2001).

${ }^{15}$ P. Samuelsson, W. Belzig, and Yu. V. Nazarov, Phys. Rev. Lett. 92, 196807 (2004).

${ }^{16}$ I. Snyman and Yu. V. Nazarov, Phys. Rev. B 77, 165118 (2008).

${ }^{17}$ Yu. V. Nazarov and M. Kindermann, Eur. Phys. J. B 35, 413 (2003).

${ }^{18}$ M. Kindermann and Yu. V. Nazarov, in Quantum Noise in Mesoscopic Physics, NATO Advanced Studies Institute, Series B: Physics, edited by Yu. V. Nazarov (Kluwer, Dordrecht, 2003), Vol. 97.

${ }^{19}$ M. Kindermann, Yu. V. Nazarov, and C. W. J. Beenakker, Phys. Rev. B 69, 035336 (2004).

${ }^{20}$ A. Di Lorenzo and Yu. V. Nazarov, Phys. Rev. Lett. 93, 046601 (2004).

${ }^{21}$ A. Di Lorenzo, G. Campagnano, and Yu. V. Nazarov, Phys. Rev. B 73, 125311 (2006).

${ }^{22}$ Yu. V. Nazarov, Superlattices Microstruct. 25, 1221 (1999).

${ }^{23}$ Yu. V. Nazarov, in Quantum Dynamics of Submicron Structures, edited by H. A. Cerdeira, B. Kramer, and G. Schön (Kluwer, Dordrecht, 1995).

${ }^{24}$ W. Belzig, in Quantum Noise in Mesoscopic Physics, NATO Advanced Studies Institute, Series B: Physics, edited by Yu. V. Nazarov (Kluwer, Dordrecht, 2003), Vol. 97.

${ }^{25}$ Yu. V. Nazarov, in Handbook of Theoretical and Computational Nanotechnology, edited by M. Rieth and W. Schommers (American Scientific, Valencia, 2006).

${ }^{26}$ W. Belzig, in CFN Lectures on Functional Nanostructures, Lec- 
ture Notes in Physics Vol. 658, edited by K. Busch, A. Powell, C. Röthig, G. Schön, and J. Weissmüller (Springer, Berlin, 2005).

${ }^{27}$ Yu. V. Nazarov and D. A. Bagrets, Phys. Rev. Lett. 88, 196801 (2002).

${ }^{28}$ B. A. Muzykantskii and D. E. Khmelnitskii, Phys. Rev. B 50, 3982 (1994).

${ }^{29}$ J. C. Cuevas and W. Belzig, Phys. Rev. Lett. 91, 187001 (2003).

${ }^{30}$ J. C. Cuevas and W. Belzig, Phys. Rev. B 70, 214512 (2004).

${ }^{31}$ G. Johansson, P. Samuelsson, and Å. Ingerman, Phys. Rev. Lett. 91, 187002 (2003).

${ }^{32}$ S. Pilgram and P. Samuelsson, Phys. Rev. Lett. 94, 086806 (2005).

${ }^{33}$ G. B. Lesovik and L. S. Levitov, Phys. Rev. Lett. 72, 538 (1994).

${ }^{34}$ M. H. Pedersen and M. Büttiker, Phys. Rev. B 58, 12993 (1998).

${ }^{35}$ L.-H. Reydellet, P. Roche, D. C. Glattli, B. Etienne, and Y. Jin, Phys. Rev. Lett. 90, 176803 (2003).

${ }^{36}$ R. J. Schoelkopf, A. A. Kozhevnikov, D. E. Prober, and M. J. Rooks, Phys. Rev. Lett. 80, 2437 (1998).

${ }^{37}$ A. A. Kozhevnikov, R. J. Schoelkopf, and D. E. Prober, Phys. Rev. Lett. 84, 3398 (2000).

${ }^{38}$ V. S. Rychkov, M. L. Polianski, and M. Büttiker, Phys. Rev. B 72, 155326 (2005).

${ }^{39}$ M. L. Polianski, P. Samuelsson, and M. Büttiker, Phys. Rev. B 72, 161302(R) (2005).

${ }^{40}$ The electron-hole excitations created by quantum pumping have been studied by M. Moskalets and M. Büttiker, Phys. Rev. B 66, 035306 (2002).

${ }^{41}$ D. A. Ivanov, H. W. Lee, and L. S. Levitov, Phys. Rev. B 56, 6839 (1997).

${ }^{42}$ H.-W. Lee and L. S. Levitov, arXiv:cond-mat/9507011 (unpublished).

${ }^{43}$ J. Keeling, I. Klich, and L. S. Levitov, Phys. Rev. Lett. 97, 116403 (2006).

${ }^{44}$ J. Keeling, A. V. Shytov, and L. S. Levitov, Phys. Rev. Lett. 101, 196404 (2008).

${ }^{45}$ G. Fève, A. Mahé, J.-M. Berroir, T. Kontos, B. Plaçais, D. C. Glattli, A. Cavanna, B. Etienne, and Y. Jin, Science 316, 1169 (2007).

${ }^{46}$ A. Mahé, F. D. Parmentier, G. Fève, J.-M. Berroir, T. Kontos, A.
Cavanna, B. Etienne, Y. Jin, D. C. Glattli, and B. Plaçais, arXiv:0809.2727 (unpublished).

${ }^{47}$ M. Moskalets, P. Samuelsson, and M. Büttiker, Phys. Rev. Lett. 100, 086601 (2008).

${ }^{48}$ S. Ol'khovskaya, J. Splettstoesser, M. Moskalets, and M. Büttiker, Phys. Rev. Lett. 101, 166802 (2008).

${ }^{49}$ M. Vanević, Yu. V. Nazarov, and W. Belzig, Phys. Rev. Lett. 99, 076601 (2007).

${ }^{50} \mathrm{Yu}$. B. Sherkunov, A. Pratap, B. Muzykantskii, and N. d'Ambrumenil, Phys. Rev. Lett. 100, 196601 (2008).

${ }^{51}$ A. G. Abanov and D. A. Ivanov, Phys. Rev. Lett. 100, 086602 (2008).

${ }^{52}$ Another derivation of the determinant formula has been given by I. Klich, in Quantum Noise in Mesoscopic Physics, NATO Advanced Studies Institute, Series B: Physics, edited by Yu. V. Nazarov (Kluwer, Dordrecht, 2003), Vol. 97; some mathematical aspects of the determinant regularization have been discussed in Ref. 41 and J. E. Avron, S. Bachmann, G. M. Graf, and I. Klich, Commun. Math. Phys. 280, 807 (2008).

${ }^{53}$ C. W. J. Beenakker, Rev. Mod. Phys. 69, 731 (1997).

${ }^{54}$ M. Vanević and W. Belzig, Europhys. Lett. 75, 604 (2006).

${ }^{55}$ The charge-transfer statistics during a finite measurement time has been studied by K. Schönhammer, Phys. Rev. B 75, 205329 (2007).

${ }^{56}$ D. Bagrets and F. Pistolesi, Phys. Rev. B 75, 165315 (2007).

${ }^{57}$ D. Bagrets and F. Pistolesi, Physica E 40, 123 (2007).

${ }^{58}$ Equation (11) coincides with Eq. (5) of Ref. 49 which has been obtained by a different method. The two terms in Eq. (5) of Ref. 49 give the same contributions, in agreement with Eq. (5) of the present paper.

${ }^{59}$ We use the following convention for the current correlation function: $S_{I}\left(t^{\prime}-t^{\prime \prime}\right)=(1 / 2)\left\langle\left\{\Delta \hat{I}\left(t^{\prime}\right), \Delta \hat{I}\left(t^{\prime \prime}\right)\right\}\right\rangle$, where $\Delta \hat{I}(t)=\hat{I}(t)-\langle\hat{I}\rangle$. The current noise power $S_{I} \equiv S_{I}(\Omega=0)$ is the zero-frequency component of the Fourier transform $S_{I}(\Omega)$.

${ }^{60}$ J. Tobiska and Yu. V. Nazarov, Phys. Rev. B 72, 235328 (2005).

${ }^{61}$ A. Di Lorenzo and Yu. V. Nazarov, Phys. Rev. Lett. 94, 210601 (2005).

${ }^{62}$ The differential noise is an odd function of $\bar{V}$ for the voltages considered in Fig. 7 and is shown for $e \bar{V}>0$ only.

${ }^{63}$ Handbook of Mathematical Functions, edited by M. Abramowitz and I. A. Stegun (Dover, New York, 1964). 